\title{
On the drying process of molded pulp products: Experiments and numerical modelling
}

Didone, Mattia; Mohanty, Sankhya; Hattel, Jesper Henri; Sonne, Mads Rostgaard; Fiordaliso, Elisabetta Maria; Checchi, Alessandro; Tosello, Guido

\section{Published in:}

Drying Technology

Link to article, DOI:

$10.1080 / 07373937.2019 .1653905$

Publication date:

2020

Document Version

Peer reviewed version

Link back to DTU Orbit

Citation (APA):

Didone, M., Mohanty, S., Hattel, J. H., Sonne, M. R., Fiordaliso, E. M., Checchi, A., \& Tosello, G. (2020). On the drying process of molded pulp products: Experiments and numerical modelling. Drying Technology, 38(12). https://doi.org/10.1080/07373937.2019.1653905

\section{General rights}

Copyright and moral rights for the publications made accessible in the public portal are retained by the authors and/or other copyright owners and it is a condition of accessing publications that users recognise and abide by the legal requirements associated with these rights.

- Users may download and print one copy of any publication from the public portal for the purpose of private study or research.

- You may not further distribute the material or use it for any profit-making activity or commercial gain

- You may freely distribute the URL identifying the publication in the public portal 


\section{On the Drying Process of Moulded Pulp Products: Experiments and}

Numerical Modelling

Mattia Didone ${ }^{a *}$, Sankhya Mohantya ${ }^{a}$, Jesper Henri Hattel ${ }^{\text {a }}$, Mads Rostgaard Sonne $^{\mathrm{a}}$, Elisabetta Maria Fiordaliso ${ }^{\mathrm{b}}$, Alessandro Checchi ${ }^{\mathrm{a}}$, Guido Tosello ${ }^{\mathrm{a}}$

${ }^{a}$ Technical University of Denmark, Department of Mechanical Engineering, $2800 \mathrm{Kgs}$. Lyngby, Denmark; ${ }^{b}$ Technical University of Denmark, Centre for Electron Nanoscopy, 2800 Kgs. Lyngby, Denmark

*Corresponding author. Email: matdid@mek.dtu.dk. ORCiD: https://orcid.org/00000002-5353-7333. 


\title{
On the Drying Process of Moulded Pulp Products: Experiments and Numerical Modelling
}

\begin{abstract}
Moulded pulp products are experiencing increased importance due to their environment-friendly characteristics with regards to both production and disposal. In this study, the thermoforming process for the production of moulded pulp products was examined by means of two experimental campaigns, with the scope of distinguishing between the water removal due to only compression (better known as cold pressing) and the water removal under additional thermal conditions. The underlying multi-physics of the processes were identified by comparison with similar manufacturing processes found in available literature, and then implemented using a 1-dimensional finite element model in COMSOL software. This fast, simple model was able to predict the water removal for a specific set of process conditions and the results were supported by corresponding experimental observations. Within a process time of up to $15 \mathrm{~s}$, temperatures ranging from $80{ }^{\circ} \mathrm{C}$ to $120{ }^{\circ} \mathrm{C}$, and a final dryness between $40 \%$ and $65 \%$, the simulations gave accurate estimates, with a difference in prediction down to $0.3 \%$. The paper thus documents the first combined experimental-simulation study for thermoforming of moulded pulp products.
\end{abstract}

Keywords: moulded pulp; thermoforming; model; simulation; drying

\section{Introduction}

In recent years, economic, environmental and social concerns have resulted in an increasing demand for sustainable packaging products. Moulded pulp is an environmentally friendly packaging material that is recyclable, compostable and potentially biodegradable, and could provide an alternative to oil-based packaging products.

The societal and industrial diffusion of the greener packaging material depends also upon the innovation of its manufacturing process. The most advanced manufacturing method in this category is thermoforming [1]. This process consists of pressing and heating a preformed product in a heated mould for a period in the order of tens of seconds. The item 
densifies and dries simultaneously, with the vapour and water being vented out via microchannels present in the mould, through the aid of vacuum suction. The resulting products have good dimensional accuracy and smooth surfaces, thus making them competitive with the plastic counterparts [2].

Nevertheless, the production of moulded pulp products is an energy and capital intensive industrial process. In particular, the cost of energy required for drying may be from eight to twenty times that needed for pre-forming [3]. Hence, research efforts are necessary in this direction for innovating the thermoforming process and making it more sustainable. In a conventional papermaking machine, three main stages can be identified: forming, pressing, and drying. In the manufacturing of moulded pulp with thermoforming, forming is carried out in a dedicated tool, while pressing and drying are combined together and occur simultaneously in the same mould. The tooling setup could vary depending upon different factors, including product shape and production rate. Heat could be applied on both halves of the mould or on a single side, and the machinery could include a rotary mechanism [4-7]. In any case, the process is essentially governed by four process parameters, namely pressure, temperature, initial solid content and time [8].

Schematically, Figure 1 displays the sequence of events taking place during a thermoforming process. Initially, the pre-formed sample is pressed while heat starts diffusing along the thickness direction. While the majority of the unbound water content is squeezed out due to volume reduction, the temperature within the remaining pulp continues to rise. At this stage, partial water evaporation is likely to occur. Afterwards, the pressure is removed from the part and the residual water content evaporates.

The aim of the present study was to examine the thermoforming process, describe the underlying physics and propose a fast simple model to predict the water removal for a specific process condition. The model would offer for instance the ability of early testing 
the dryness efficiency of a particular tooling setup. It could also help in scoping the combination of process parameters that could lead to the highest drying rate. Overall, understanding and simulating the thermoforming of moulded pulp can lead to the increase of the process efficiency.

The literature currently available does not address specifically any model for the drying of moulded pulp products, and the work presented in this article is the first of its kind. Anyway, the phenomena occurring during the drying step and the process conditions are similar in nature to two paper-drying technologies that have been extensively studied: hot pressing and impulse drying. Therefore, in Section 3.1, the underlying physics of the drying process is described by appropriately referencing the literature.

A comprehensive review related to the development of conventional paper drying technologies between the periods 2000 - 2018 can be found in the review article by Stenström [9]. 


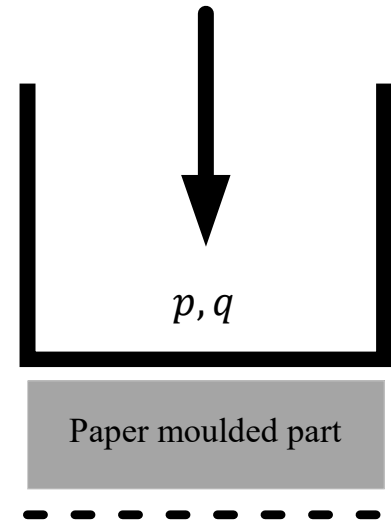

Support and water drainage

Initial compression and heat transfer.
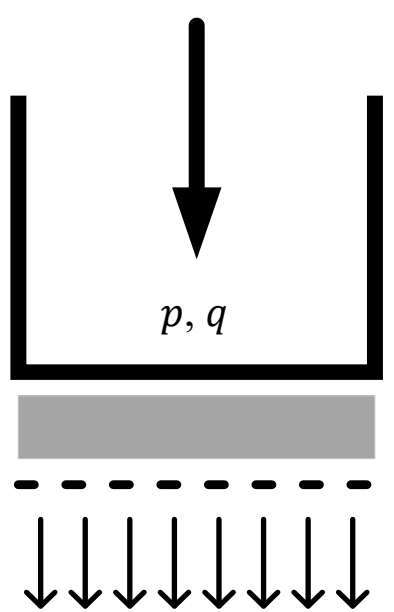

Wet pressing stage. Temperature continues to rise and eventual water evaporation.

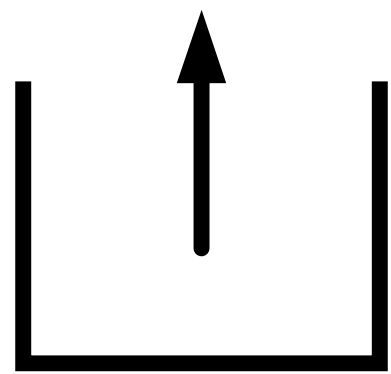

(Steam)

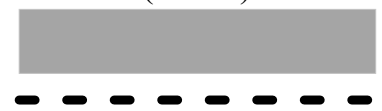

Post-compression stage. Eventual residual water evaporation.

Figure 1: Schematics of the thermoforming of moulded pulp.

\section{Experiments}

The experiments presented in this section were fundamental in order to describe the underlying physics of a thermoforming process, and for the calibration and validation of the model.

With the goal of isolating the effect of the heat applied on the water removal, two experimental campaigns were designed and conducted.

In the first campaign, pre-formed samples were cold-pressed, while suction via vacuum $(0.01 \mathrm{MPa})$ was active on the side opposite to the pressing element. The experiment resembled in essence a wet pressing process with the addition of the vacuum suction. The process parameters under control in this experiment were: pressure and process time. The quantities measured were: initial, final and bone dry weight of the samples; initial and final samples thickness. Subsequently, a performance indicator named dryness efficiency $\left(D_{e}\right)$ was introduced and calculated in order to assess the amount of water removed. The 
thickness reduction $\left(\mathrm{THK}_{\text {red }}\right)$ was also calculated to evaluate the relative dimensional change in the sample's cross-section.

In the second experimental campaign, the pressing element was heated thus fully resembling a thermoforming process. The heat was applied on the top part of the sample, while suction via vacuum $(0.01 \mathrm{MPa})$ was applied at the bottom throughout the whole process duration. In this experiment, the temperatures development in the top and bottom centre part of the samples was also recorded. This will be consequently used to support the description of the underlying process physics.

The process parameters under control in this second experiment were: pressure, temperature and process time. The quantities measured were: initial, final and bone dry weight of the samples; initial and final samples thickness; temperature development at the top and bottom centre of the samples throughout the whole process. As per before, the performance indicators dryness efficiency and thickness reduction were calculated.

\section{Methods}

When referring to moulded pulp, one could imagine a product with a geometry that presents a combination of flat surfaces and edges. In this study however, the samples geometry was a disk with a diameter of $200 \mathrm{~mm}$, a pre-formed thickness around $4 \mathrm{~mm}$ and a final thickness in the dried state around $1.8 \mathrm{~mm}$. In any case, the model presented is able to predict the dryness of the disks with respect to the process parameters, and it is therefore applicable to a more complex geometry presenting sidewalls and edges.

Throughout the experiments, the pulp material used was made of a mixture of commercially available chemical pulps. Unfortunately, further and detailed information about the pulp composition cannot be disclosed, as they are of proprietary nature of one of the project's partners. 
The moulded pulp disks were pre-formed with a custom-made sheet former that resembles the "TAPPI T205" test method [10], as can be seen in Figure 2. The moulded disks were pre-formed to an average solid content of $20 \%$ to $25 \%$. Once dried, the samples had a basis weight around $600 \mathrm{~g} / \mathrm{m}^{2}$.

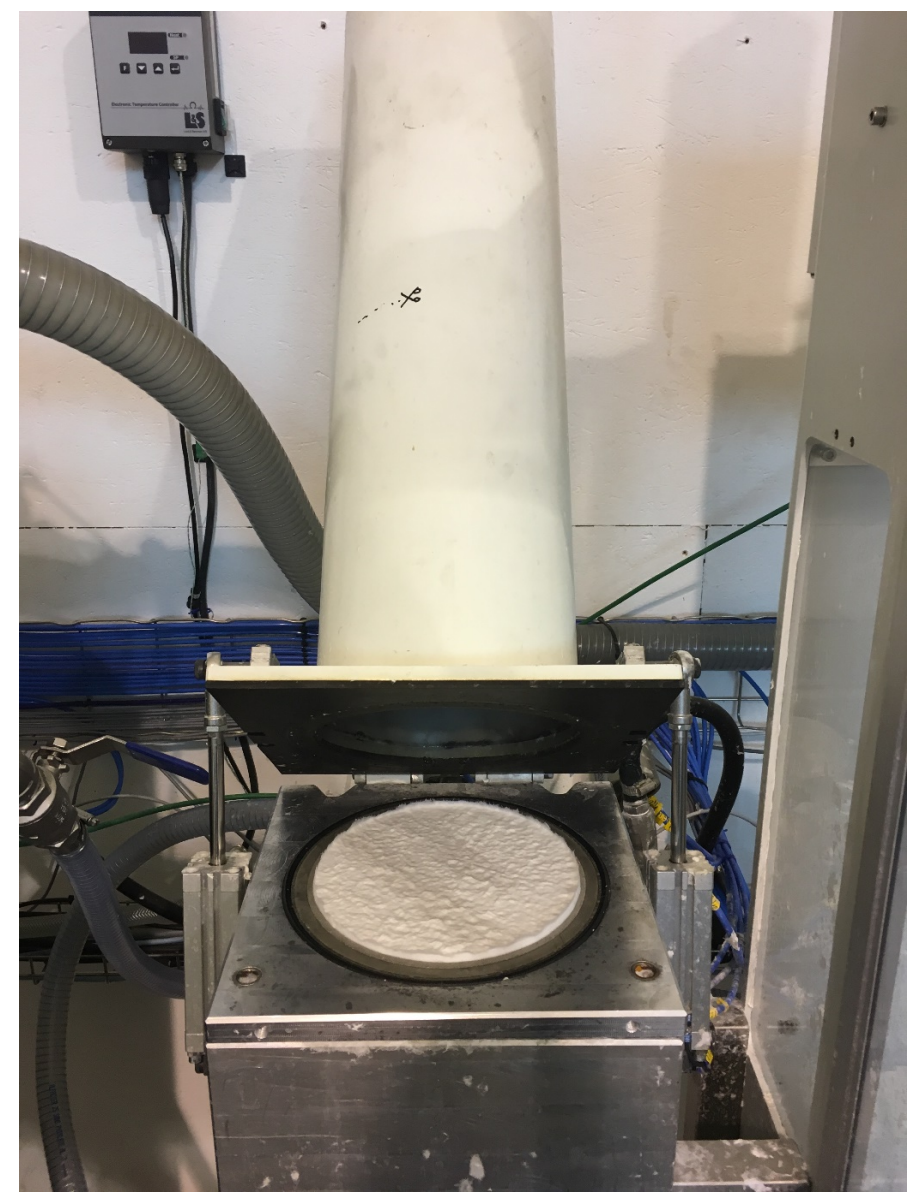

Figure 2: Custom-made sheet former used to pre-form the moulded pulp samples.

Figure 3 shows the laboratory-scale thermoforming machine employed in the experimental part of this work. The piston that can be seen in the upper part of Figure 3 had two purposes: pressing the samples by descending towards them and heating them at the same time. The piston, in fact, contained a flat heater with a diameter of $125 \mathrm{~mm}$, a power output of $700 \mathrm{~W}$, and a maximum working temperature around $350{ }^{\circ} \mathrm{C}$. However, 
due to heat losses in the piston, the effective temperature on the outer surface of the piston was considerably below $350{ }^{\circ} \mathrm{C}$. A hydraulic motor pump unit drove the piston.

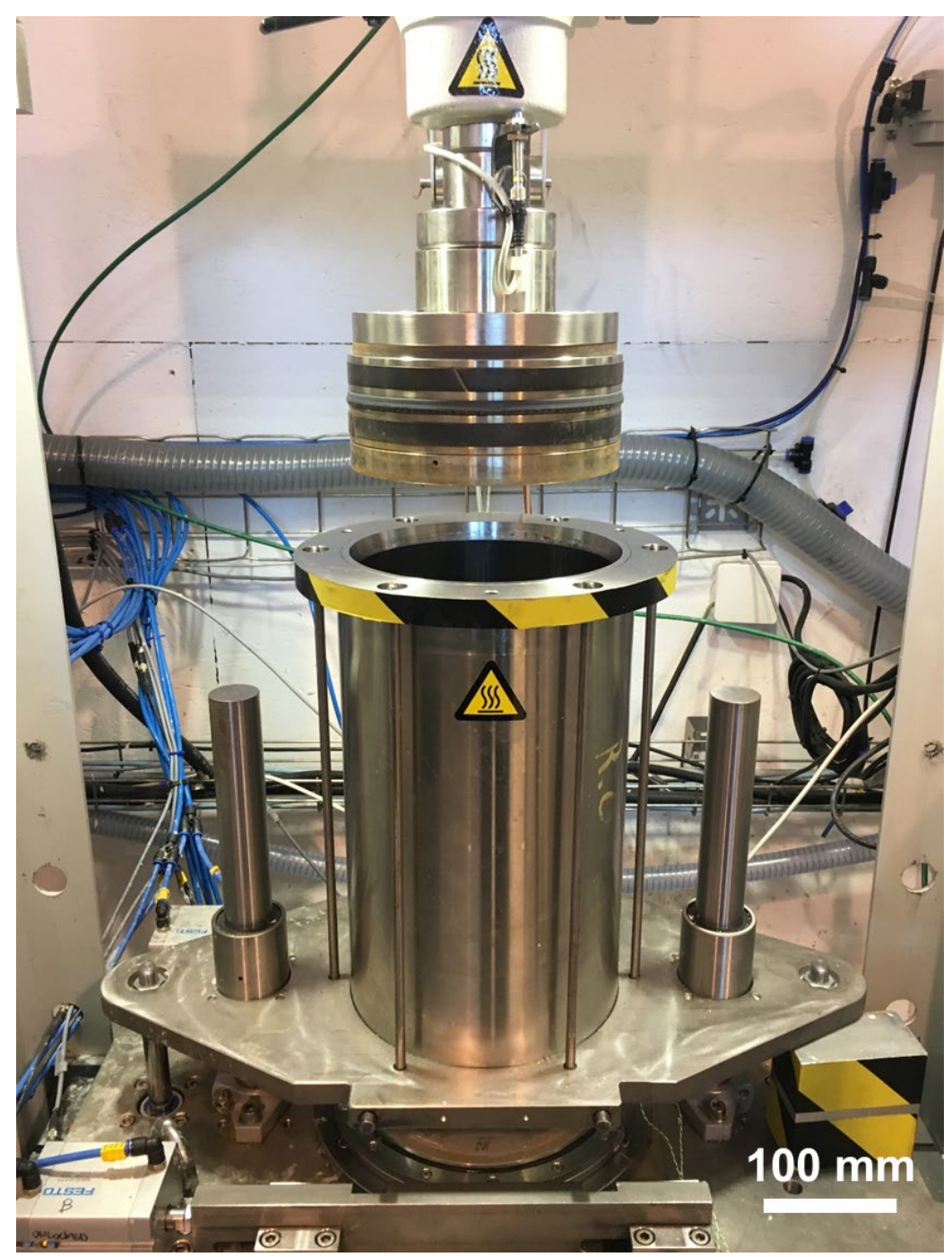

Figure 3: The laboratory-scale thermoforming machine. The picture shows the pressing and heating element at the top and, at the very bottom, is where the samples were placed, on top of a metal mesh.

The pre-formed moulded samples were placed at the bottom of the laboratory-scale thermoforming machine, on top of a fine metal wire mesh. Underneath it, a rigid metal screen filter disk gave support to the metal wire mesh. This arrangement (as shown in 
Figure 4) laid on top of the base support, below which suction via vacuum was operated for venting the water/steam in excess.

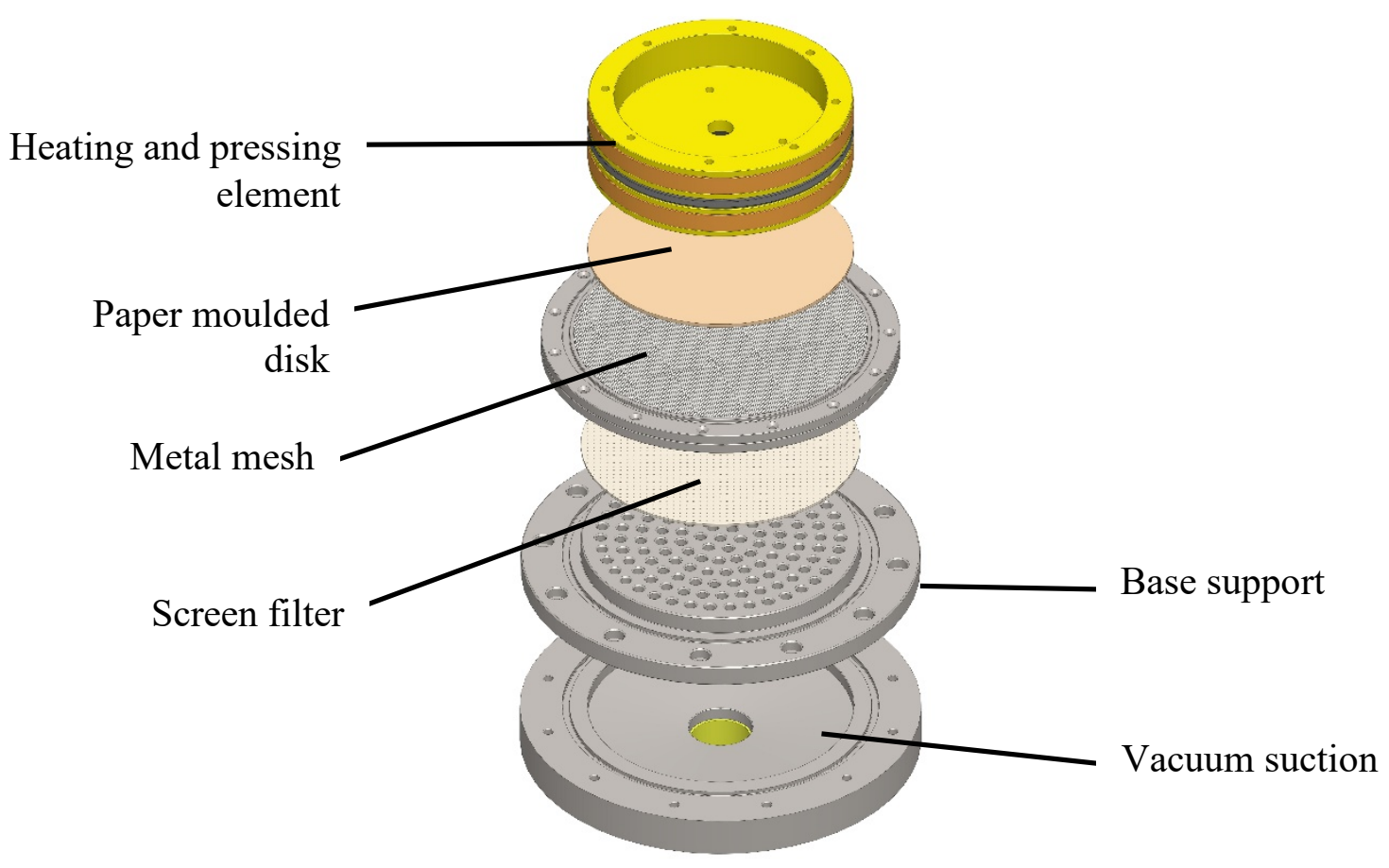

Figure 4: Scheme of the water evacuation system.

Throughout this work, it was assumed that the piston exerted a uniform pressure onto the samples' surface. This assumption was justified by the diameter and distribution of the holes of the different elements that were part of the water evacuation system. The sizes are reported in Table 1. In particular, the holes of the metal mesh were smaller than the average fibres length of the pulp employed. Therefore, the moulded pulp samples were well supported and the pressing force acting on them could be assumed to be uniformly distributed on the entire surface. 
Table 1: Holes diameter and distance between two consecutive holes for the elements forming part of the water evacuation system.

\begin{tabular}{|c|c|c|}
\hline Element & $\begin{array}{c}\text { Nominal holes diameter } \\
(\mathbf{m m})\end{array}$ & $\begin{array}{c}\text { Nominal distance } \\
\text { between holes } \mathbf{( m m})\end{array}$ \\
\hline Metal mesh & 0.3 & 0.2 \\
\hline Screen filter disk & 1.0 & 7.1 \\
\hline Base support & 10.0 & 15.0 \\
\hline
\end{tabular}

The machine was operated as follows. After a pre-formed sample was placed in position, the piston moved towards it with a relative slow velocity $(45 \mathrm{~mm} / \mathrm{s})$. Its descending trajectory continued until, after coming in contact with the sample, the compressive force exerted by the piston reached the imposed pressure limit. Immediately after, suction via vacuum was applied underneath the sample, and remained operative for the whole duration of the cycle. While the sample was pressed, heat was also transferred via conduction from the external surface of the piston to the moulded sample, along the thickness direction. The process continued for the imposed value of process time. Once the process time elapsed, the piston moved upwards towards its resting position, thus releasing the pressing force that was acting on the sample. Later, the sample was collected and measured.

In the cold pressing experiments, the parameters pressure and process time were varied according to Table 2. The experimental setup corresponding to the pressure level of 0.3 MPa was replicated three times for assessing the standard deviation, while the other three pressure levels were replicated only one time, resulting in 20 experiments. The process time was varied up to $600 \mathrm{~s}$ in order to verify and reaffirm the validity of the “Decreasing Permeability (DP) Model” [11], introduced later in Section 3.

Table 2: Process parameters settings for the cold pressing process investigation.

\begin{tabular}{|c|c|c|}
\hline Parameter & Unit & Levels \\
\hline Pressure & $\mathrm{MPa}$ & $0.3 ; 0.6 ; 1.0 ; 1.3$ \\
\hline Process time & $\mathrm{s}$ & $3 ; 300 ; 600$ \\
\hline
\end{tabular}


In the thermoforming experiments, the parameters pressure, process time and temperature were varied, according to Table 3 , for a total of 36 experiments. Process time in this case was set with the purpose of resembling an optimal industrial case.

Table 3: Process parameters settings for the thermoforming process investigation.

\begin{tabular}{|c|c|c|}
\hline Parameter & Unit & Levels \\
\hline Pressure & $\mathrm{MPa}$ & $0.3 ; 0.6 ; 1.0 ; 1.3$ \\
\hline Process time & $\mathrm{S}$ & $3 ; 9 ; 15$ \\
\hline Temperature & ${ }^{\circ} \mathrm{C}$ & $80 ; 120 ; 160$ \\
\hline
\end{tabular}

It is important to remark that suction via vacuum was active underneath the sample in both the experimental campaigns, for the whole duration of the process cycle. The vacuum was set to $0.01 \mathrm{MPa}$.

Two quantities were measured in both the cold pressing and the thermoforming experiments, namely the sample weight and thickness. In the thermoforming experiments, the temperature development in the middle-top and bottom part of the sample were also measured. In order to quantify the water removed in the moulding process, a performance indicator named dryness efficiency $\left(D_{e}\right)$ was proposed as follows:

$$
D_{e}=\frac{m_{\text {in }}-m_{\text {out }}}{m_{\text {in }}-m_{f}} \%
$$

Where:

$m_{\text {in }} \quad$ is the initial sample weight, expressed in $\mathrm{g}$

$m_{\text {out }}$ is the mass of the sample right after the molding process, expressed in $g$

$m_{f} \quad$ is the mass of the fibers, i.e. the bone dried weight of the sample, expressed in $g$ 
$D_{e}$ takes into account the initial water content of the sample, which could present a large variation. It gives an immediate impression of the effectiveness at which water was removed for a specific set of process parameters. $D_{e}$ could vary from $0 \%$ to $100 \%$, where $100 \%$ corresponds to a complete removal of the water content. The parameter name contains the word "efficiency" because it represents a proportion between the actual amount of water removed in a specific process condition (numerator), and the ideal situation (denominator), which corresponds to a complete removal of water.

Dryness efficiency differs from the usual definition of consistency $(S)$, widely used in the conventional paper industry, in which the initial paper mass $\left(m_{i n}\right)$ is not taken into account. In fact, consistency reads as follows (according to [12]):

$$
S=\frac{m_{f}}{m_{\text {out }}}
$$

Where:

$m_{f} \quad$ is the mass of the fibers, i.e. the bone dried weight of the sample, expressed in $g$ $m_{\text {out }}$ is the mass of the sample right after the drying process, expressed in $\mathrm{g}$

The samples were weighted with a compact scale with a capacity of $200 \mathrm{~g}$ and a readability of $0.01 \mathrm{~g}$ (EK-200i, Buch \& Holm A/S, Herlev, Denmark). The thickness was measured using an analog gauge (Dial Gauge 8336-24, Hexagon Metrology AB, Stockholm, Sweden) mounted on an appropriate support, as shown in Figure 5. The thickness was determined in four different points marked on the samples' surface (Figure 6). The thickness was measured both before and after the thermoforming process. 


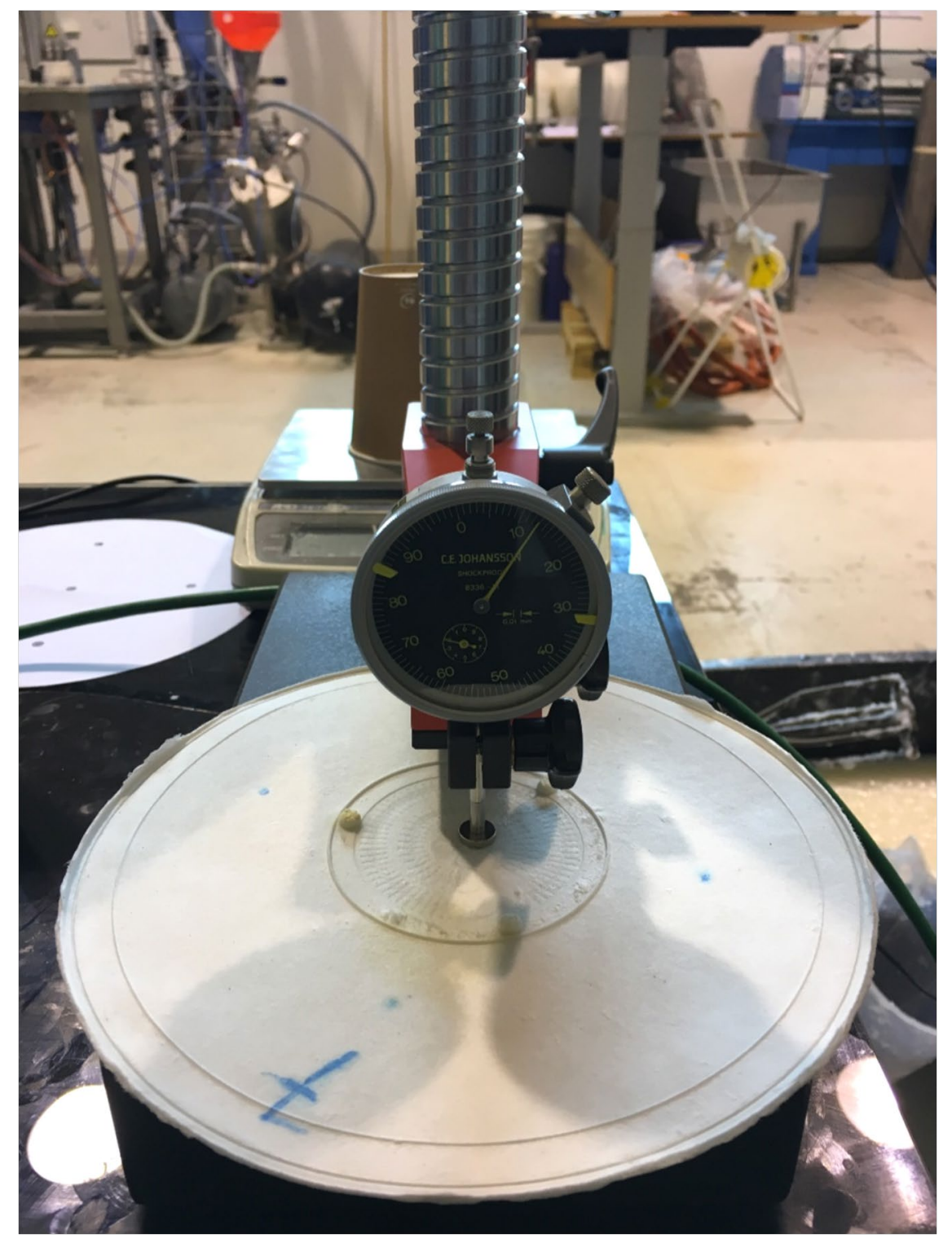

Figure 5: Photo of the analog gauge "Dial Gauge 8336-24" mounted on an appropriate support.

The gauge was used to measure the samples' thickness variation during the experiments.

In order to evaluate the relative thickness reduction, the performance indicator thickness reduction $\left(T H K_{\text {red }}\right)$ was defined as follows:

$$
T H K_{\text {red }}=\frac{T H K_{\text {in }}-T H K_{\text {out }}}{T H K_{\text {in }}} \%
$$

Where: 
THK $K_{\text {in }} \quad$ is the initial sample thickness, i.e. the thickness of the pre-formed sample, expressed in $\mathrm{mm}$

THK out is the final sample thickness, i.e. the thickness of the sample after the wetpressing/thermoforming process, expressed in $\mathrm{mm}$

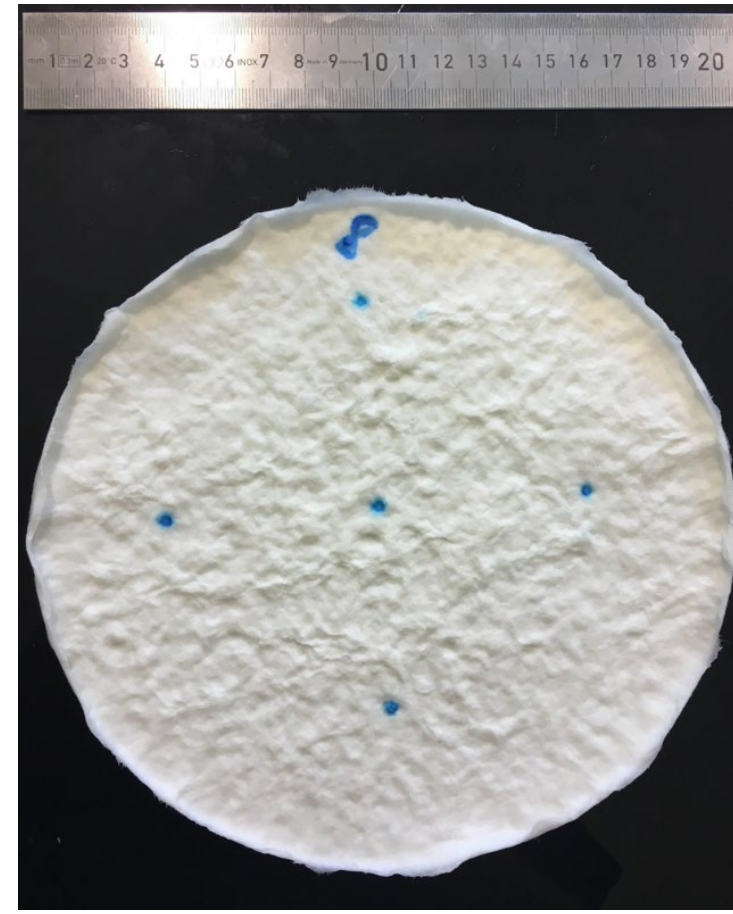

(a)

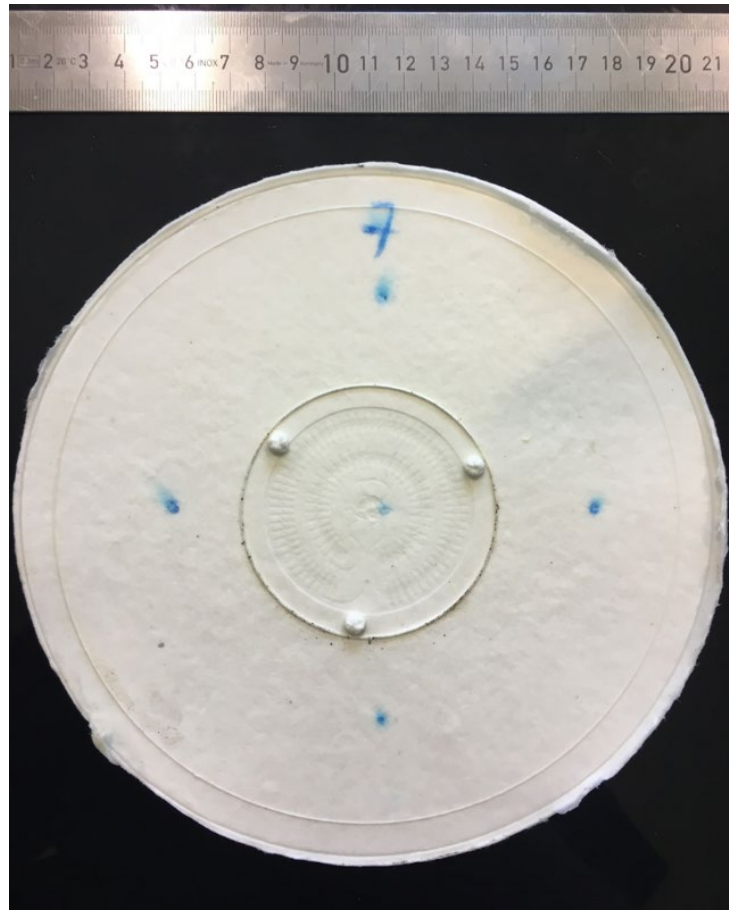

(b)

Figure 6: (a) Pre-formed paper moulded disk. (b) Thermoformed sample. The four external blue dots are the points where the thickness was measured.

During the thermoforming experiments, temperatures in the middle-top and middlebottom part of the sample were recorded. The top temperature was acquired with a custom-made temperature sensor positioned directly on the external surface of the piston (MesoScribe $^{\mathrm{TM}}$, Central Islip (NY), United States). The custom-made sensor consists of an array of thermocouples integrated in the piston and in direct contact with the pulp. The interface between the pulp and the sensor array consists of a black paint layer of few micrometres. 
The mid-bottom temperature was measured with two K-type thermocouples, with a probe diameter of $0.075 \mathrm{~mm}$ (RS Components, Corby, United Kingdom), as shown in Figure 7. Both the custom-made sensor and thermocouples were interfaced with a dedicated data acquisition hardware, in order to acquire the signals synchronously. Data was logged with the software LabVIEW. Data acquisition was sampled at a frequency of $10 \mathrm{~Hz}$.

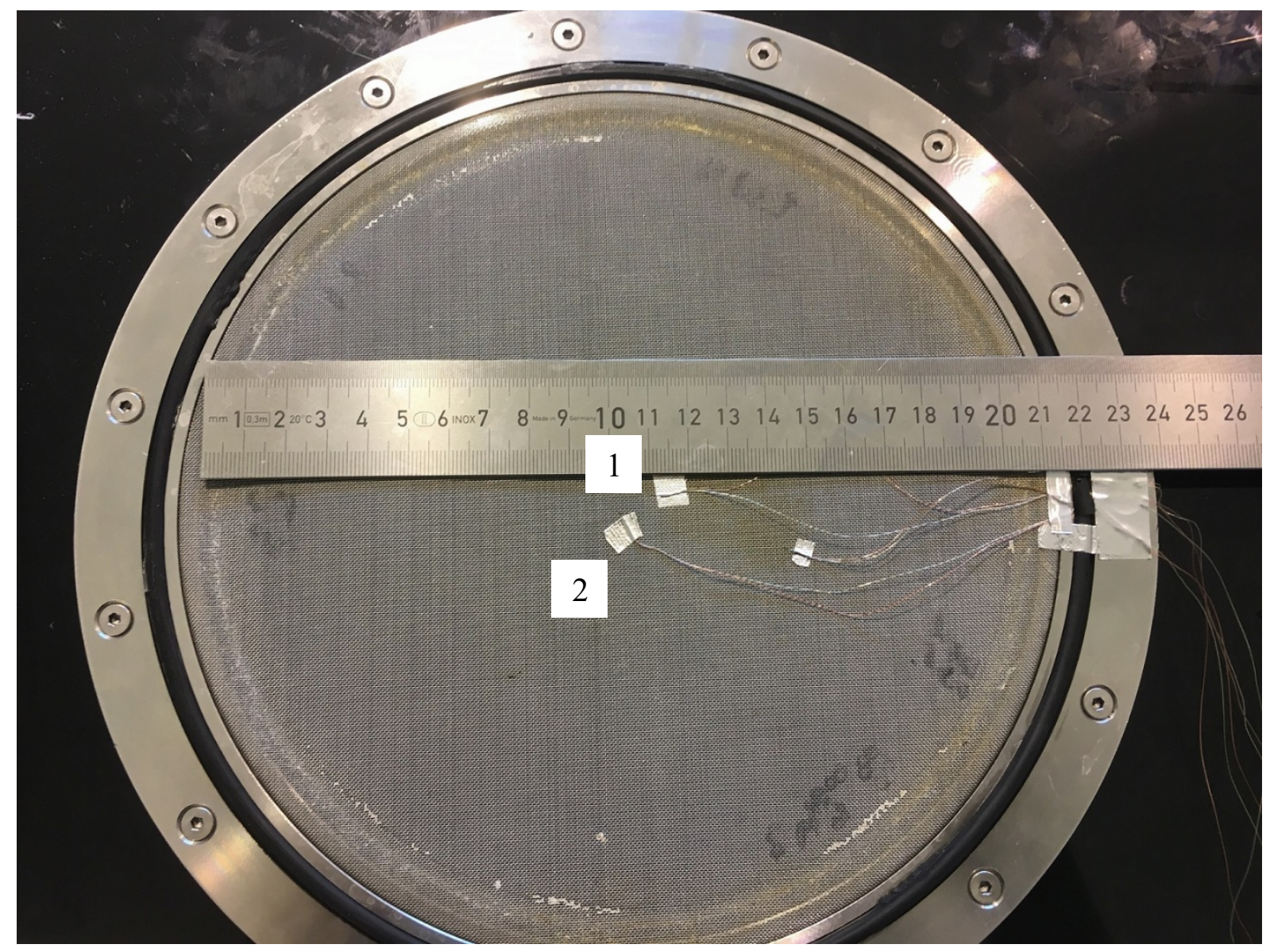

Figure 7: Photo of the two K-type thermocouples (number 1 and 2 in the picture) used to measure the temperature at the bottom of the samples.

For the sake of clarity, the procedure followed during the experiments is given below:

i) A moulded paper sample was formed to an average initial solid content of $20 \%$ to $25 \%$. 
ii) The piston was heated to the set level of temperature (only in the case of a thermoforming experiment).

iii) The pre-formed sample was placed in position in the laboratory-scale thermoforming machine. The piston started descending until the pressing force applied onto the sample reached the set level of pressure.

iv) Suction via vacuum was activated underneath the sample, while heat transferred from the piston's surface to the top of the sample via conduction. This stage lasted for the set duration of the process time.

After the process time elapsed, the piston travelled upwards, thus releasing the pressing force. The sample was collected and the measurements were carried out.

\section{Experimental Results}

\section{Cold-pressing Experiments}

Figure 8 summarizes the results obtained from the cold-pressing experiments where the dryness efficiency as a function of increasing pressure and process time is shown. Data was fitted with a two-parameter logarithmic function of the type:

$$
y=b \cdot \ln (x \cdot a)
$$

Fitted values for $a$ and $b$ coefficients are also given in Figure 8. The predicted adjusted R-Square values are above 0.9 , indicating an excellent fit of the logarithmic function. Although at first glance, the plotted data points suggest that a linear function fit could be adequate, the choice of a logarithmic fitting was motivated by similar trends found in literature [11].

State-of-the-art cold-press sections are able to achieve web solid contents in excess of $50 \%$, which are resembled by the blue curve in Figure 8 . Higher solids contents, over $70 \%$, are reached in the case that the process is carried out for $300 \mathrm{~s}$ and $600 \mathrm{~s}$. Moreover, 
the difference between the two curves is minor, showing the tendency that a limiting consistency is reached, regardless of how long a pressing force is applied [13].

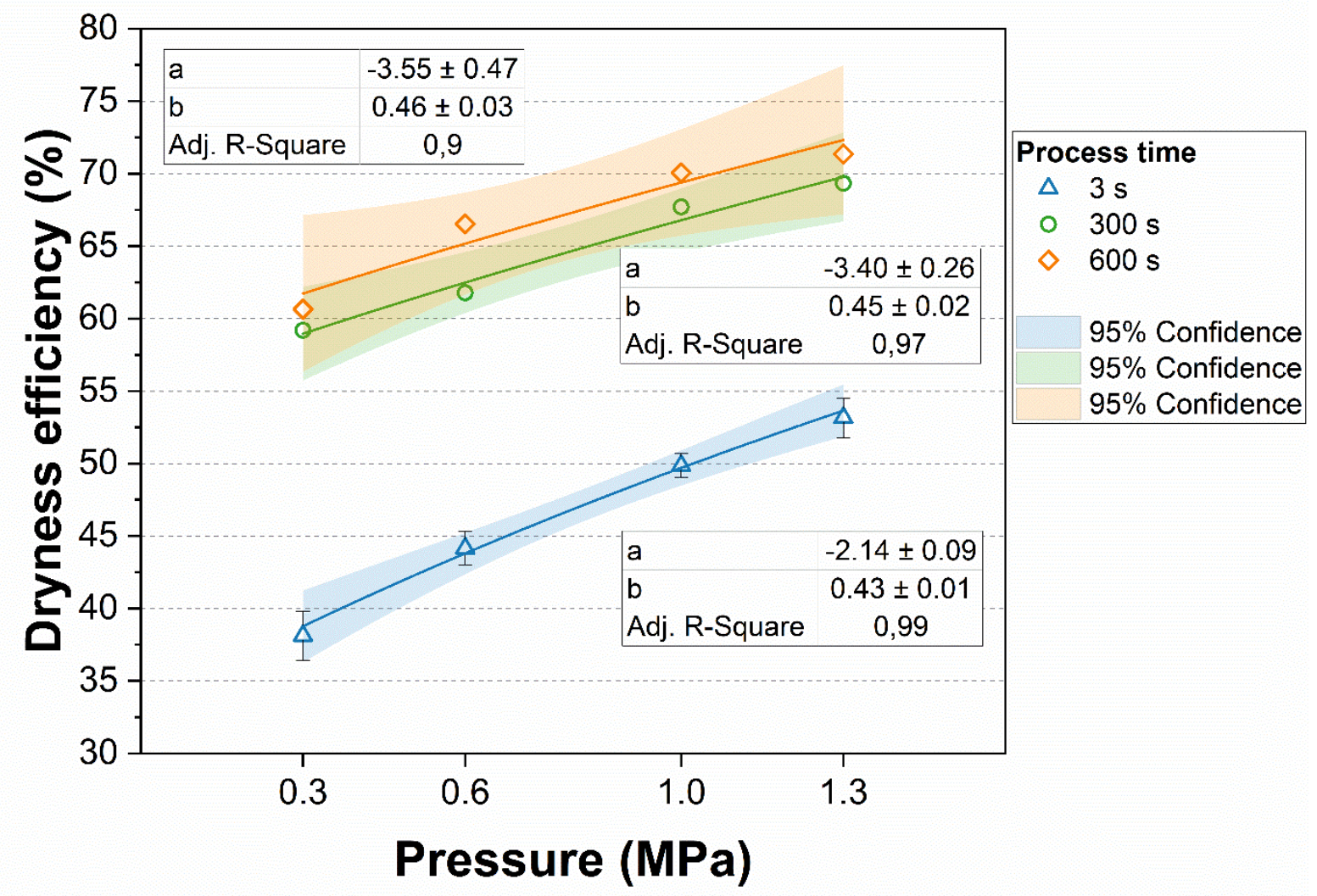

Figure 8: Dryness efficiency as a function of increasing pressure and process time for the wet pressing experiments. The results were fitted with a two-parameter logarithmic function. The $95 \%$ confidence bands of the fitting functions are also shown.

\section{Thermoforming Experiments}

The results from the thermoforming experiments are set out in Figure 9 where the dryness efficiency with respect to increasing pressure, process time and temperature is reported. Data was also fitted with equation (4), and the resulting $95 \%$ confidence bands are also shown. Unlike the cold-pressing experiments, the confidence intervals of the logarithmic fit corresponds to a large range of dryness efficiency (as large as $\sim 20 \%$ ), highlighting the 
added influence of the thermal conditions on the drying and justifying the need of developing a physics-based model for the thermo-forming process.

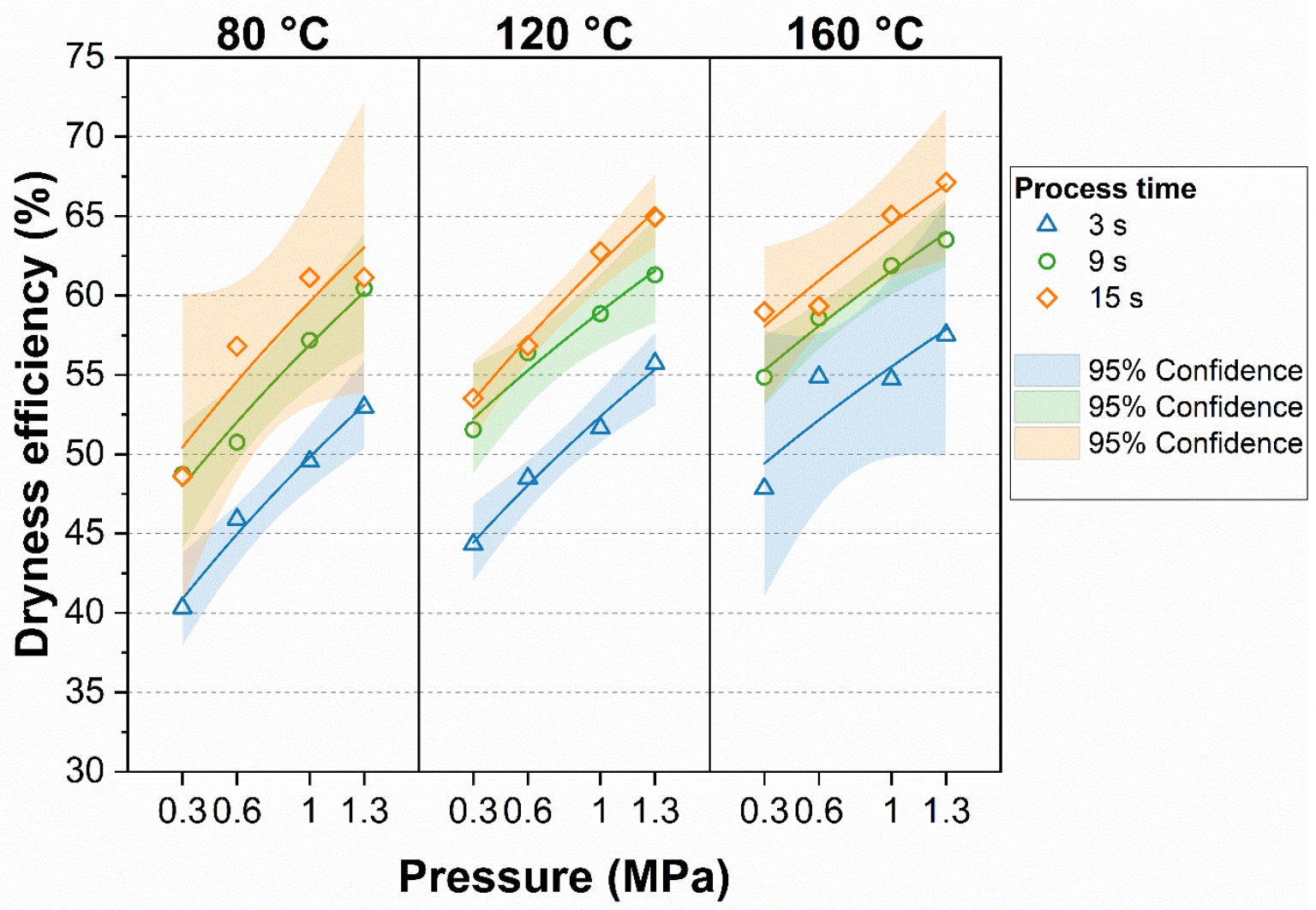

Figure 9: Dryness efficiency as a function of increasing pressure, temperature and process time for the thermoforming experiments. The results were fitted with a two-parameter logarithmic function. The $95 \%$ confidence bands of the fitting functions are also shown.

\section{Temperature Measurements during a Thermoforming Process}

The measurement of the temperature development over time during a thermoforming event is reported in Figure 11. The measurements were carried out under the following process conditions: pressure at $1.3 \mathrm{MPa}$, temperature of $160{ }^{\circ} \mathrm{C}$, process time of $15 \mathrm{~s}$. The blue line represents the temperature recorded by the top sensor, while the orange and green lines are the temperatures recorded by the two thermocouples placed underneath the sample. 
An explanation for the events corresponding to the points marked on the blue line from "i" to "iv" follows here:

i) The sensor, which is embedded in the piston, is recording the initial piston temperature, which is around $165^{\circ} \mathrm{C}$. As soon as the piston comes in contact with the pre-formed sample, initially at ambient temperature, the temperature drops suddenly down to about $130^{\circ} \mathrm{C}$.

ii) An insulating vapour layer is likely to form between the hot piston's surface and the still cold pre-formed moulded pulp similar to the Leidenfrost effect [14]. Correspondingly, a slight temperature increase is measured by the sensor, which is probably re-gaining heat from the hot piston.

iii) Once the vapour layer dissipates, the piston temperature starts decreasing again as heat is transferred to the sample.

iv) The piston is lifted from the sample, and the sensor is gaining heat from the hot source, and so the temperature starts rising again. 


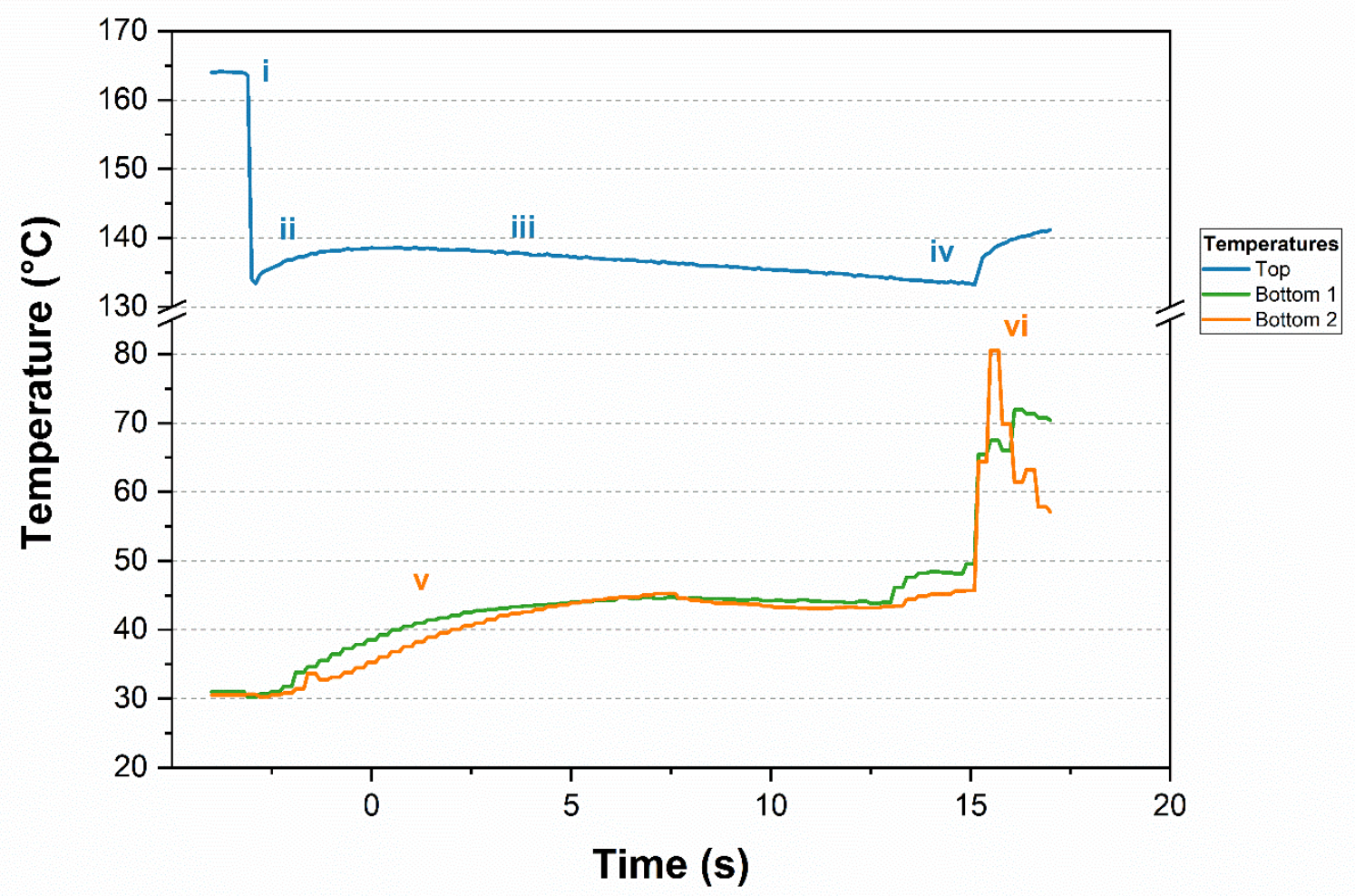

Figure 10: Temperatures development during a thermoforming event. The blue line represents the temperature acquired at the top centre surface of the sample, while the green and orange lines are the temperatures at the bottom.

The green and orange lines in Figure 11 correspond to the thermocouples on the moulded paper pulp, and exhibit a temperature increase over time due to the heat transfer from the hot piston to the pre-formed sample (point "v"). Point "iv" corresponds to the piston being lifted from the sample and the pressing force that was acting onto it being released. The water content held by and within the fibres in the upper part of the sample, closer to the heat source, is likely to be in a superheated state, given the applied pressure and temperature. Immediately after the pressing force is discharged, steam forms, expands and moves towards the colder bottom part of the sample. Consequently, a sudden increase in temperature (point "vi") is registered by the thermocouples. Similar evidences of postnip steam expansions are reported in literature $[15,16]$ concerning the hot pressing and impulse drying technology. 
Relation between Thickness Reduction and Dryness Efficiency

The relative thickness reduction of the samples during the cold pressing and thermoforming experiments were assessed. Figure 13 depicts the relation observed during the cold-pressing investigation, while Figure 14 reports one of the thermoforming experiments. In the latter case, the samples dried at $160{ }^{\circ} \mathrm{C}$ presented defects in form of blisters and internal delamination, corroborating the post-nip steam expansion hypothesis. An example of the defects is shown in Figure 15. Therefore, it was not possible to accurately measure the final samples' thickness, and calculate the thickness reduction for the corresponding process condition.

The horizontal error bars reported in the two graphs represent the combined standard deviation $\left(\sigma_{c}\right)$ for the computation of the performance indicator thickness reduction. The value of thickness was measured in four points and the combined standard deviation was evaluated according to the following equation [17]:

$$
\sigma_{c}(y)=\sqrt{\sum_{i=1}^{N}\left(\frac{\partial f}{\partial x_{i}}\right)^{2} \sigma^{2}\left(x_{i}\right)}
$$

Where:

$f \quad$ is the considered function, in this case thickness reduction

$x_{i} \quad$ represents each of the function terms 


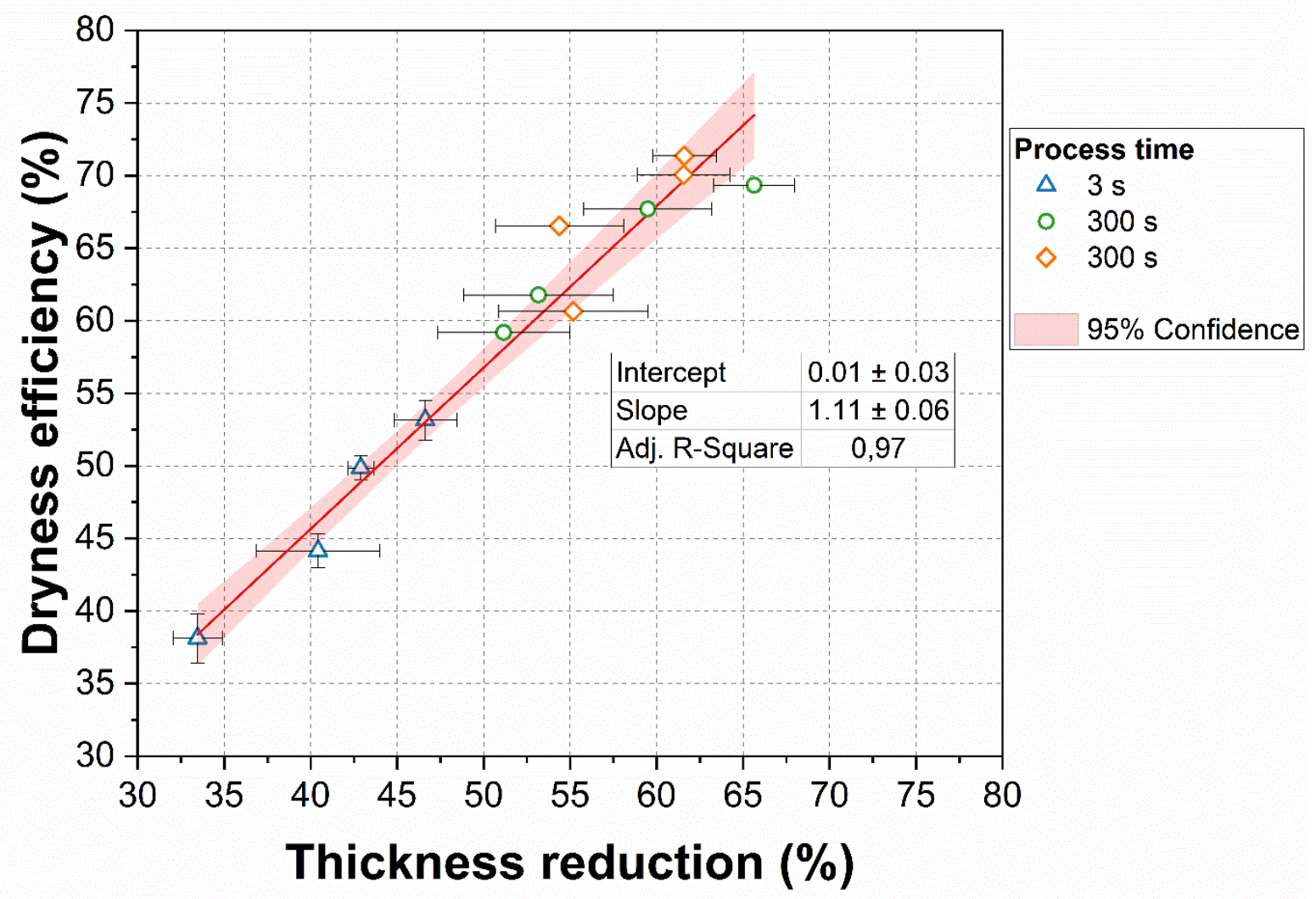

Figure 11: Dryness efficiency as a function of thickness reduction in a wet pressing event. The linear data-fitting and the corresponding $95 \%$ confidence band are also reported.

It can be readily observed that Figure 11 and Figure 12 share a similar linear trend. The trends were fitted with a linear equation of the type reported in Equation (6). Consequently, the two fitted lines were compared in Figure 14, where the contribution of the process parameters is also highlighted.

$$
y=a+b \cdot x
$$

where $a, b$ are the two coefficients to be fitted with the available data. The similar trends observed between the two indicators suggest the possibility of using the thickness reduction as an on-line measurable indicator for the efficiency of the thermo-forming process while dryness efficiency will correspond to an offline post-process indicator. 


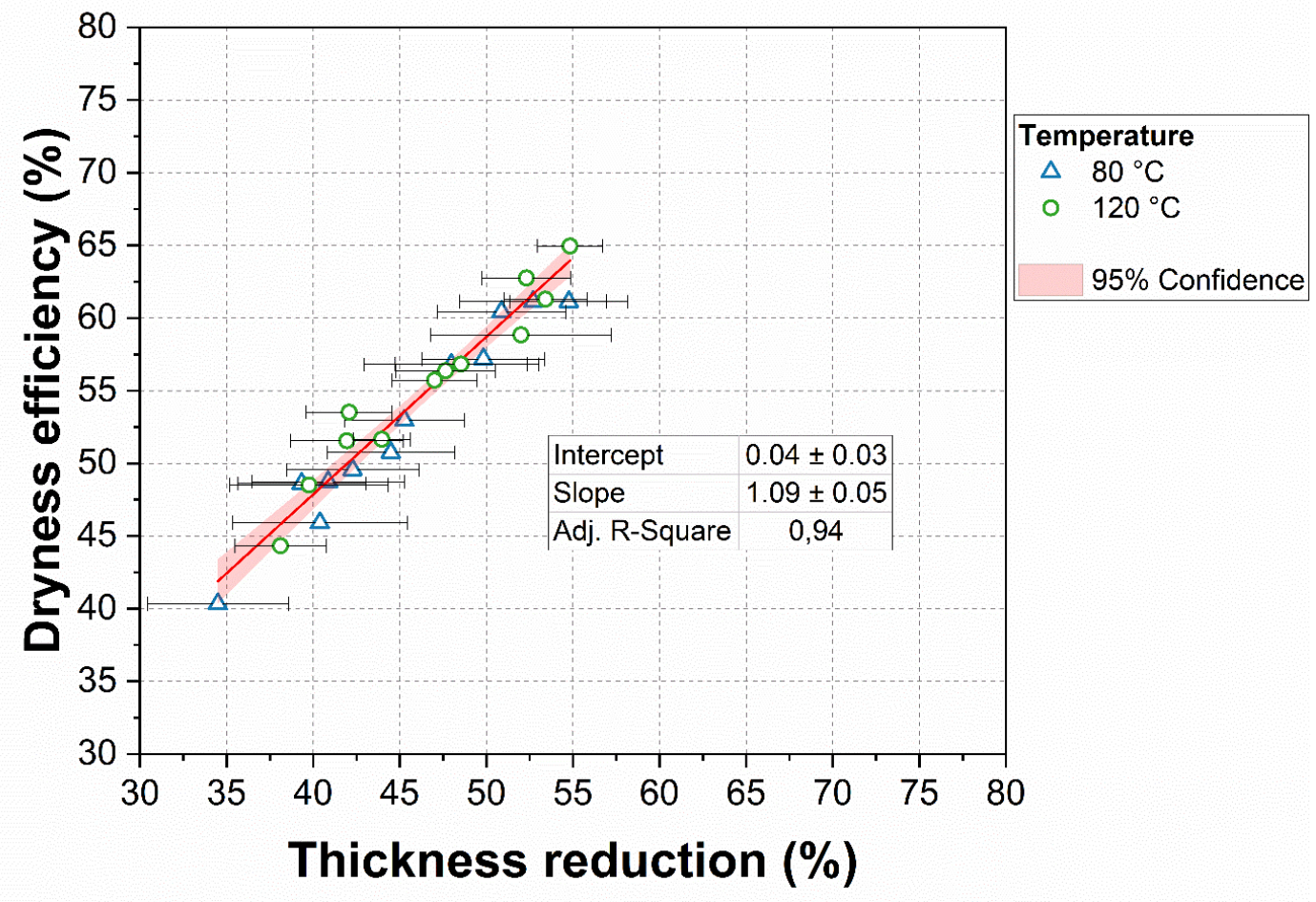

Figure 12: Dryness efficiency as a function of thickness reduction in a thermoforming event. The linear data-fitting and the corresponding $95 \%$ confidence band are also reported.

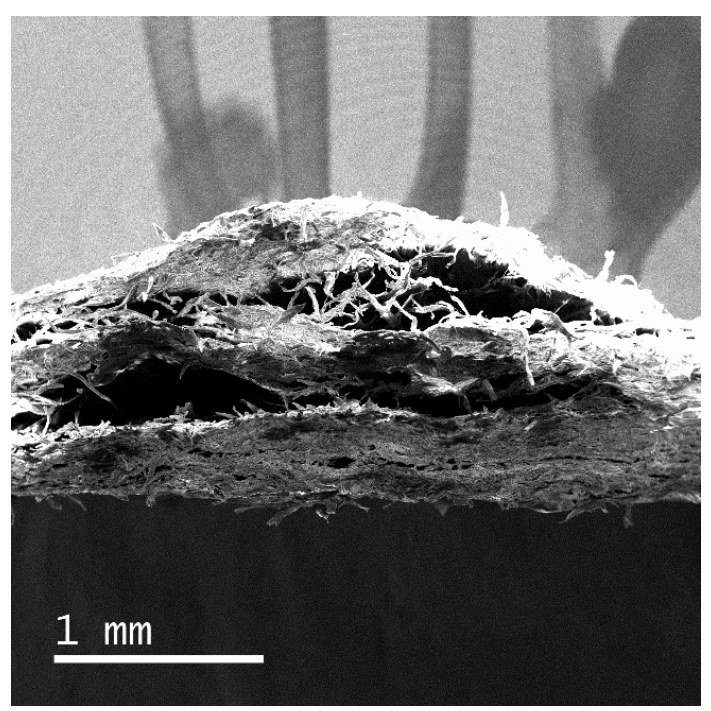

(a)

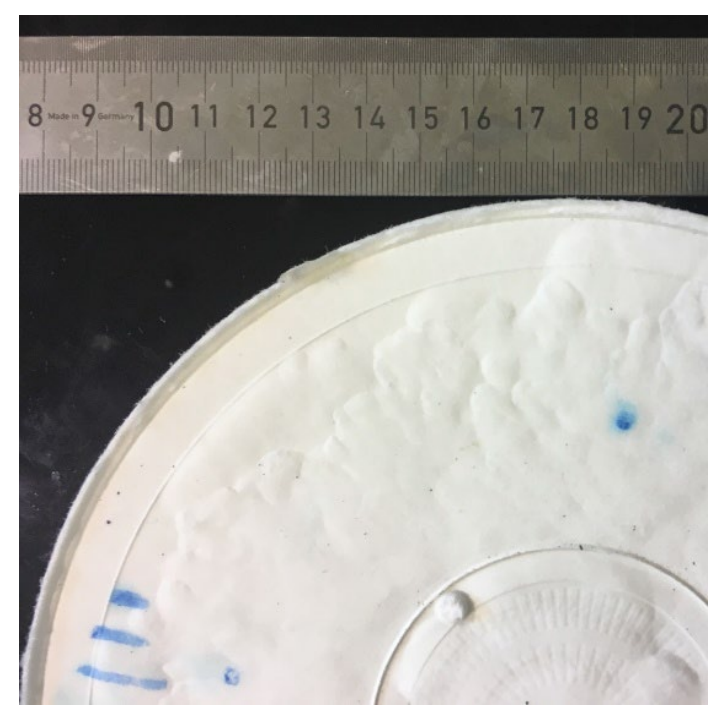

(b)

Figure 13: Evidence of delamination for the samples thermoformed at $160{ }^{\circ} \mathrm{C}$. (a) SEM image of a delaminated cross section. (b) Blisters/internal delamination evidence on the sample's surface. 


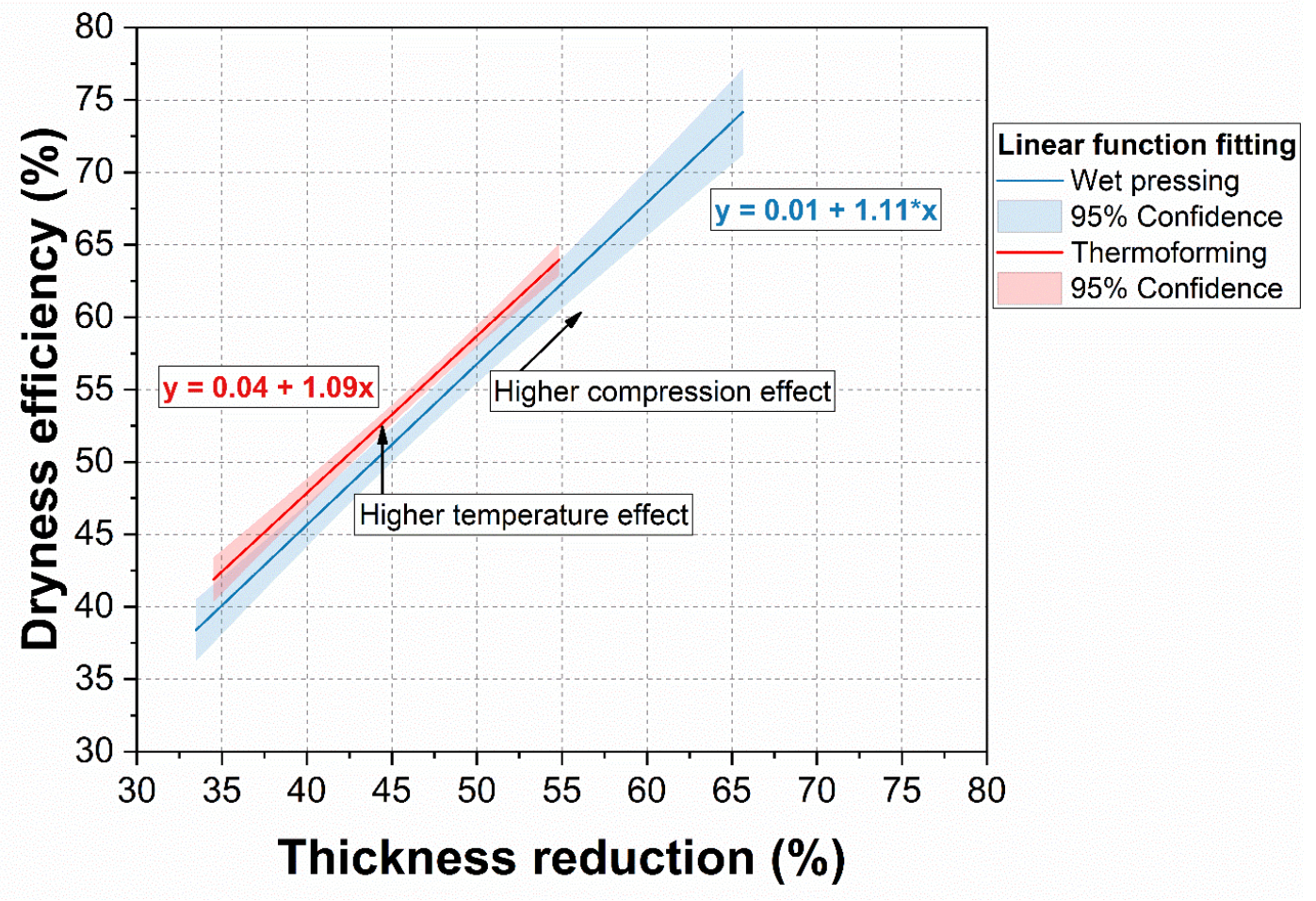

Figure 14: Comparison of the two linear fitted functions of the wet pressing and the thermoforming experiments.

\section{Numerical Model}

This section comprises a description of the physical phenomena occurring during a thermoforming process, which contribute to the removal of water. Further, a set of model equations, the corresponding boundary conditions and definition of material properties are presented.

\section{Underlying Physics}

In explaining the underlying physics, each aspect contributing to the removal of water is analysed separately. The description accounts for the thermomechanical behaviour over time of the moulded pulp material. The material is considered as a three-phase medium, constituted of wood fibres, water and gas filled pores, where water exists in a bound and unbound form. The unbound or free water exists in spaces between fibres or in large pores 
(diameter greater than $25 \mu \mathrm{m}$ ) within the fibres. Bound water, instead, exists in smaller fibre pores and is chemically adhered to the fibres.

The main phenomena responsible for the water transport and discharge are:

i) volume reduction;

ii) vacuum dewatering;

iii) heat transfer and water phase transition to vapour;

iv) post-pressing evaporation and heat pipe phenomena.

\section{Volume Reduction}

Volume reduction, named also "thickening" [18], refers to the continuous collapse of the pulp fibre network under the application of a pressing load. The compression occurs along the thickness direction and is due to a reduction in void volume. The applied stress is mainly balanced by two components, viz. the hydraulic pressure and the fibre network stress (often referred to as structural pressure) [19]. In a wet sheet compression, it was actually shown that the dominant effect offering resistance to deformation is the structural pressure [20].

The dewatering process can be described as a two-phase flow problem in which a solid matrix is compressed forcing the interstitial fluid to diffuse out of the domain. Models of dewatering are typically posed in a one spatial dimension, with the application of a fixed load or a fixed rate compression [21].

At the fibre level, the compression of the pulp network in the thickness direction can be conceived to occur in three intervals. First, the void volume is reduced and water from the larger pores flows out. At a later stage (when the compression rate is close to zero), the pulp deforms due to elasto-plastic buckling of the fibre wall. In this interval, water from the smaller pores is removed from the domain. When the fibres have collapsed, 
further deformation is achieved by the "crushing" of the fibres [22]. As water is expelled from the fibre web, its permeability decreases.

Wet pressing also considerably changes the pulp density distribution. Especially, in this case where water removal is fast and one-sided, the density on the side next to the pressing element may increase by as much as one-third, and yet remain unchanged on the other [23]. Fibres twist, collapse, and gap closures are the major mechanisms in such a pulp structure densification. Fibres twist so that their cross-sectional axis becomes parallel with respect to the moulding plane. Assuming that the moulded pulp product has a layered structure, fibres in each layer are twisted and eventually collapsed in wet pressing, which reduces the thickness of each layer. Twisting and collapsing reduce the amount of space taken by the fibres. The total reduction in the paper thickness is the sum of the thickness reduction of each layer [24].

\section{Vacuum Dewatering}

Suction via vacuum is active throughout the thermoforming process and its main intent is to evacuate water, thus avoiding re-wetting the sample. Moreover, it contributes in creating a pressure gradient across the thickness direction. The hydraulic pressure is in fact highest on the surface in contact with the pressing element, and lowest on the surface exposed to the vacuum. The sum of the hydraulic and structural pressures must be constant at each z-direction point of the sample due to equilibrium, i.e. if there is a hydraulic pressure gradient in the web there is also a z-direction gradient in the structural pressure [23]. The resulting pressure level is fundamental in determining the eventual water to vapour phase transition. 


\section{Heat Transfer and Water Phase Transition to Vapour}

The heat transfer leads to an increase in temperature of the sample. As in the case of the pressure gradient, it is fair to assume a temperature gradient along the thickness direction. The increasing of temperature leads to an enhanced dewatering due to thermal softening of the fibres and a decreasing of the water viscosity. Back [25] explains the thermal softening, i.e. the decrease of the Young modulus of the fibrous network, by the reduced inter-fibre and intra-fibre hydrogen bonding. A second effect of temperature is to change the surface tension of water thereby affecting the capillary pressure of water meniscus in the pores. The amount of pressure required before the liquid meniscus can be moved is then lowered $[8,18,26]$. This also leads to improved consolidation.

During a thermoforming process, the water content at a specific z-coordinate of the sample have a definite value of hydraulic pressure and temperature. Consequently, as soon as the critical moisture content is reached, evaporation takes place [27].

\section{Post-Pressing Evaporation and Heat Pipe}

Figure 11 shows an intense heat transfer process occurring when the pressing element is lifted from the paper sample, which most probably involves vaporization and condensation within the decompressed sheet. Such a process is usually termed a heat pipe process. The vapour flows from the top surface towards the bottom (colder portion) of the sample and condenses, producing a rapid rise of the temperature. Similar evidences were reported in [28].

\section{Models for Involved Physical Phenomena}

\section{Empirical Wet Pressing Model}

Wet pressing has been modelled in literature as a process of forcing water from a porous medium in which resistance to water flow increase with decreasing water content. Models 
based on hydraulic forces alone are often better able to account for experimental observations. The "Decreasing Permeability (DP) Model" [11], first introduced in 1991, is the most complete model of this form.

An early version of the DP model was derived from the Kozeny-Carman equation [29]. It was based on the assumption that pressure acts on water throughout the web, whether directly or through fibre walls, and that permeability and flow path length have a nonlinear dependence on the moisture content of the web (see equation 7).

$$
m=m_{0}\left(1+\frac{A_{p} n m_{0}^{n} I}{v W^{2}}\right)^{-\frac{1}{n}}
$$

Where:

$m_{0} \quad$ is the initial moisture content

$A_{p} \quad$ is the specific permeability, expressed in $\mathrm{g} / \mathrm{m}$

$I \quad$ is the press impulse, expressed in $\mathrm{kPa} \mathrm{s}$

$n \quad$ is the compressibility factor

$W \quad$ is the basis weight, expressed in $\mathrm{kg} / \mathrm{m}^{2}$

$v \quad$ is the kinematic viscosity, expressed in $\mathrm{m}^{2} / \mathrm{s}$

When considering pressing to a high solids content, permeability reaches values close to zero. McDonald and Kerekes [30] accounted for this condition by including a limiting (or equilibrium) moisture content, $m_{e}$. The equilibrium moisture depends upon furnish type and pressure. Incorporating this effect into equation (7) gives the final form for the equation for predicting wet pressing over the entire range of moisture content:

$$
m=\left(m_{0}-m_{e}\right)\left(1+\frac{A_{p} n\left(m_{0}-m_{e}\right)^{n} I}{v W^{2}}\right)^{-\frac{1}{n}}
$$


Where:

$m_{e} \quad$ is the limiting moisture at which permeability in effect reached zero

Such a simple analytical model provides a good approximation against which numerical simulation implementations of a poro-elasticity model could be verified.

\section{Heat Transfer in Porous Media}

Heat transfer by conduction is macroscopically described by the Fourier's law [31].

$$
q=-k A \frac{\partial T}{\partial x}
$$

Where:

$q \quad$ is the diffusive heat flow perpendicular to the surface of area $A$, expressed in $\mathrm{W}$

$A \quad$ is the area of the surface, expressed in $\mathrm{m}^{2}$

$k \quad$ is the thermal conductivity, expressed in $\mathrm{W} /(\mathrm{m} \mathrm{K})$

$T \quad$ is the temperature, expressed in ${ }^{\circ} \mathrm{C}$

$x \quad$ is the space parameter perpendicular to the surface, expressed in $\mathrm{m}$

In a wet capillary-porous media, conductivity can be described by partly arranging in parallel and partly in series the main conducting components (fibres and water) [32]. The idea was firstly presented by Palosaari in 1976 (see equation 10) [33].

$$
\begin{gathered}
k_{\text {par }}(T, p)=k_{f} \varepsilon_{f}+k_{w}(T, p) \varepsilon_{w} \\
\frac{1}{k_{\text {ser }}(T, p)}=\frac{1}{\frac{\varepsilon_{f}}{k_{f}}+\frac{\varepsilon_{w}}{k_{w}(T, p)}} \\
k_{\text {eff }}(T, p)=\frac{1}{\frac{1-a}{k_{\text {ser }}}+\frac{a}{k_{\text {par }}}}
\end{gathered}
$$

Where: 
$k_{\text {par }}(T, p) \quad$ is the parallel thermal conductivity, expressed in $\mathrm{W} /(\mathrm{m} \mathrm{K})$

$k_{f} \quad$ is the fibre thermal conductivity, expressed in $\mathrm{W} /(\mathrm{m} \mathrm{K})$

$\varepsilon_{f} \quad$ is the fibre volume fraction

$k_{w}(T, p) \quad$ is the water thermal conductivity, expressed in $\mathrm{W} /(\mathrm{m} \mathrm{K})$

$\varepsilon_{w} \quad$ is the water volume fraction, complementary of $\varepsilon_{f}$

$k_{\text {ser }}(T, p) \quad$ is the series thermal conductivity, expressed in $\mathrm{W} /(\mathrm{m} \mathrm{K})$

$a$

is an empirical parameter, set equal to 0.46 [33]

With the appropriate finite element formulation of the standard conductive-advective heat transfer equation (see equation 11), combined with an equivalent porous-media conductivity from equation (10), it is possible to predict the temperature evolution in time and space within the considered domain. However, the flow of water within the domain would necessitate further modelling to account for the advection terms in the heat transfer equations.

$$
\left(\rho C_{p}\right)_{e f f} \frac{\partial T}{\partial t}+\rho C_{p}(u \cdot \nabla T)=\nabla \cdot\left(k_{e f f} \nabla T\right)-\dot{m}_{\text {evap }} L_{\text {evap }}
$$

Where:

$\rho \quad$ is the density, expressed in $\mathrm{kg} / \mathrm{m}^{3}$

$C_{p} \quad$ is the specific heat capacity, expressed in $\mathrm{J} /(\mathrm{kg} \mathrm{K})$

$\dot{m}_{\text {evap }}$ is the specific moisture evaporation rate, expressed in $\mathrm{kg} /\left(\mathrm{m}^{3} \mathrm{~s}\right)$

$L_{\text {evap }}$ is the latent heat of vaporization, expressed in $\mathrm{J} / \mathrm{kg}$

$u \quad$ is the velocity of the fluid (water) in the porous medium, expressed in $\mathrm{m} / \mathrm{s}$

\section{Fluid Flow in Porous Media}

The flow of a fluid through a porous medium under a pressure gradient typically corresponds to a creeping flow that can be described using Darcy's law [34]. 


$$
u=-\frac{\kappa_{p}}{\mu} \nabla p
$$

Where:

$\kappa_{p} \quad$ is the permeability of the porous medium, expressed in $\mathrm{m}^{2}$

$\mu \quad$ is the dynamic viscosity of water, expressed in $\mathrm{kg} /(\mathrm{m} \mathrm{s})$

$p \quad$ is the pressure within the porous medium, expressed in $\mathrm{Pa}$

For flow under high-pressure gradients and typically with Reynolds number greater than one, inertial effects also become relevant. The corresponding non-linear behaviour is then modelled through the Forchheimer equation instead [35], which relates the pressure gradient to the flow rate through the inertial permeability $k_{i}$.

$$
\nabla p=-\frac{\mu}{\kappa_{p}} u-\frac{\rho_{w a t e r}}{\kappa_{i}} u^{2}
$$

Where:

$\rho_{\text {water }} \quad$ is the water density, expressed in $\mathrm{kg} / \mathrm{m}^{3}$

$\kappa_{i} . \quad$ is the flow inertial permeability, expressed in $\mathrm{m}^{2}$

To calculate the pressure gradient driving the flow, the Darcy law is typically combined together with the continuity equation:

$$
\frac{\partial\left(\epsilon_{p} \rho_{\text {water }}\right)}{\partial t}-\nabla \cdot\left(\rho_{\text {water }} u\right)=-\dot{m}_{\text {evap }}
$$

Where

$\epsilon_{p} \quad$ is the porosity of the pulp, expressed in dimensionless numbers

By combining the resulting temperature and pressure gradient levels within the domain, it is possible to identify whether the conditions for water evaporation are met. 
Consequently, it is possible to calculate the dryness efficiency for a specific set of initial and boundary conditions.

\section{Current Model Implementation}

While a detailed description of the physics underlying a thermoforming process has been provided in section 1.2, modelling requires simplifications and assumptions. For instance, a 3D poroelastic compression simulation of the moulded pulp could provide accurate predictions for the thickness reduction and the water removal, but would be relatively computationally intensive and would require several accurate inputs (e.g. initial porosity, water concentration, fibre material strength, etc.) that can be difficult to provide.

For the current modelling study, only the flow in the vertical (thickness) direction was considered relevant, thus obtaining a one-dimensional domain. The paper pulp was described as a saturated porous medium, i.e. a two-phase system, containing wood fibres and water. Adhering to the different phenomena in the physical dewatering process, the simulation assumed that the total mass of water removed corresponded to a combination of direct dewatering due to compression (proportional to the thickness reduction), dewatering due to evaporation from the bottom surface, and dewatering due to the flow of water through the porous medium under high compression (the previously explained Darcy flow). The water loss due to post-pressing evaporation was neglected as well. Thus,

$$
m_{\text {out }}=m_{\text {thickness reduction }}+m_{\text {evaporation }}+m_{\text {Darcy flow }}
$$

The dewatering due to thickness reduction was calculated based on the initial moisture content, the initial weight and the bone-dry weight of the sample, along with the equation:

$$
m_{\text {thickness reduction }}=C 1 * \rho_{\text {water }} * A_{\text {disk }} * t_{\text {in }} *\left(1-\left(\frac{T H K_{\text {out }}}{T H K_{\text {in }}}\right)\right)
$$

Where: 
$A_{\text {disk }} \quad$ is the disk area, expressed in $\mathrm{m}^{2}$

THK $\quad$ is the initial thickness of the sample, expressed in $\mathrm{m}$

THK $\quad$ is the thickness of the compressed sample, expressed in $\mathrm{m}$

C1 is a constant of proportionality derived using the cold-pressing experimental results at $0.3 \mathrm{MPa}$ for $3 \mathrm{~s}$ pressing duration

The dewatering due to evaporation and flow through a porous medium is a coupled process, as the effective evaporation rate is proportional to the pressure and temperature at the bottom surface. Heat is transferred by both unsteady heat conduction in the thickness direction as well as by advection by Darcy flow. Thus, the top surface of the moulded pulp domain was subjected to a heat flux from the steel press maintained at a constant temperature while the bottom surface of the sample was exposed to ambient temperatures. While most evaporation could be considered to occur closer to the exposed bottom surface, the potential for evaporation through the sample sides exist and was accounted for by introducing a corresponding mass source/sink.

For calculating the flow through the porous medium, local pressure equilibrium between the fluid and the solid phase was also considered. The permeability of the paper pulp was assumed to be proportional to the porosity in the sample, and thus the current problem corresponded to Darcy flow under a gradient permeability problem. The desaturation of the pulp was neglected in the current model for the Darcy flow calculations (otherwise requiring more complex models), but was indirectly considered through a dependence of permeability on the effective water concentration within the sample (similar to the Decreasing Permeability Model discussed before). Rewetting was also neglected, justified from the presence of vacuum (pressure of $0.01 \mathrm{MPa}$ ) that removed the water leaving the domain. The water and solid (pulp) domains were also assumed intrinsically 
incompressible for the flow calculations - thus limiting the time within which the simulations were representative of the real thermo-forming process.

The effect of internal viscous stresses in the fluid phase and the contact conductance between the heating element and the paper sample was neglected, as were the local temperature differences between the water and the paper phase - the assumption being justified by the relatively small domain considered, which is of $2 \mathrm{~mm}$ in height (thickness direction).

The material properties used for the simulations can be found in Table 4 .

Table 4: Material properties.

\begin{tabular}{|c|c|c|}
\hline Parameter & Unit & Value \\
\hline $\begin{array}{c}\text { Specific moisture capacity } \\
\text { of paper pulp }\end{array}$ & - & 0.003 \\
\hline $\begin{array}{c}\text { Moisture conductivity of } \\
\text { paper pulp }\end{array}$ & $\mathrm{kg} /(\mathrm{m} \mathrm{s})$ & $1.29 \mathrm{e}-9$ \\
\hline $\begin{array}{c}\text { Moisture mass transfer } \\
\text { coefficient }\end{array}$ & $\mathrm{m} / \mathrm{s}$ & $3.638 \mathrm{e}-7$ \\
\hline Density of paper pulp & $\mathrm{W} /\left(\mathrm{m} \mathrm{K}^{3}\right)$ & $1550[36]$ \\
\hline $\begin{array}{c}\text { Thermal conductivity of } \\
\text { paper pulp }\end{array}$ & $\mathrm{J} /(\mathrm{kg} \mathrm{K})$ & $0.335[36]$ \\
\hline $\begin{array}{c}\text { Specific heat capacity of } \\
\text { paper pulp }\end{array}$ & $\mathrm{W} /\left(\mathrm{m}^{2} \mathrm{~K}\right)$ & $1340[36]$ \\
\hline Heat transfer coefficient & $\mathrm{m}^{2}$ & $0.3 \mathrm{e}-12$ \\
\hline $\begin{array}{c}\text { Permeability coefficient } \\
\text { for paper pulp }\end{array}$ & & 25 \\
\hline
\end{tabular}

The numerical models were implemented in the commercial software COMSOL Multiphysics ${ }^{\circledR}$ along with in-house MATLAB scripts, with the latter being primarily used for computing the water concentration and in model calibration. The heat transfer in solids, Darcy flow and transfer of diluted species modules of COMSOL Multiphysics ${ }^{\circledR}$ were relevant for the current implementation. Figure 17 shows the coupling between the different sub-models resulting in the computation of water concentration. The $1 \mathrm{D}$ domain was discretized into a uniform grid of 400 nodes, and the same grid was used for all the 
sub-models (schematics shown in Figure 18). The computations were performed on a 6core mobile workstation using the default Multifrontal Massively Parallel sparse direct Solver (MUMPS) algorithm and resulted in a computation time of $5 \mathrm{~s}$ for simulating $3 \mathrm{~s}$ of thermoforming \& 15 mins for simulating $600 \mathrm{~s}$ (10 mins) of thermoforming.

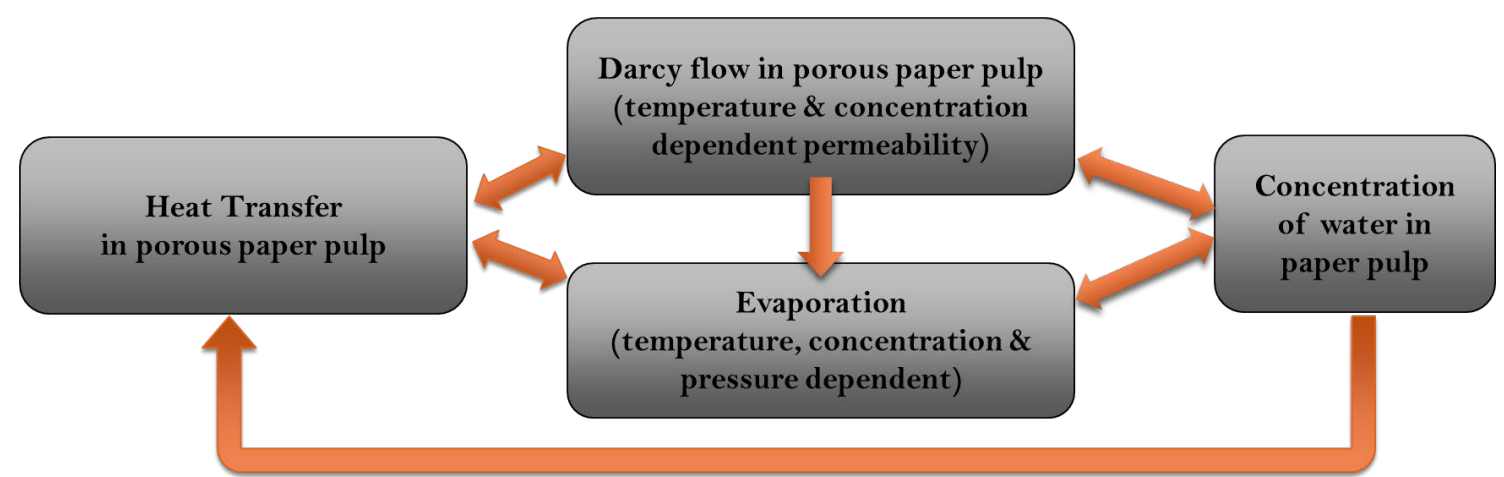

Figure 15: Coupled multi-physics model for drying of moulded pulp products. Note the one-way coupling between the Darcy-flow \& the evaporation models and the concentration calculations \& the heat transfer models.

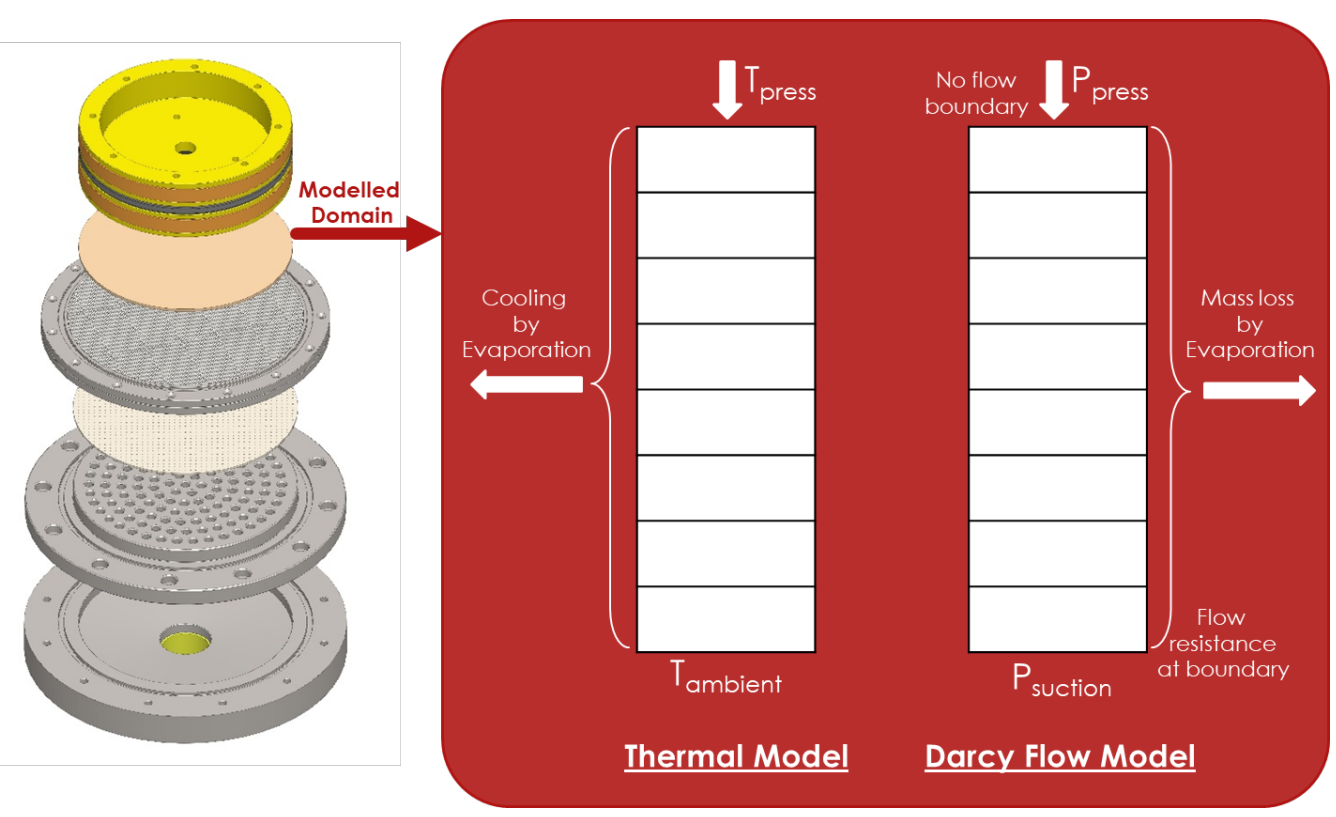

Figure 16: Schematics of lumped 1D model used for simulating thermo-forming of moulded pulp showing the modelled domain \& the corresponding thermal and flow boundary conditions. 


\section{Numerical Solution \& Model Validation}

The dewatering under cold pressing was first simulated by assuming the temperature to be at a constant value of $20^{\circ} \mathrm{C}$. Even under these conditions, due to the vacuum applied on one side of the domain, i.e. $0.01 \mathrm{MPa}$, the evaporation rate increases resulting in a nonnegligible water loss. As mentioned earlier, the experimental results corresponding to 0.3 MPa pressure and $3 \mathrm{~s}$ time duration were used to calibrate the model.

Figure 19 shows the simulated concentration gradient of water within the sample at times $3 \mathrm{~s}, 300 \mathrm{~s}$ and $600 \mathrm{~s}$. As described previously, the water concentration (or density) is higher closer to the top surface (left edge in the figure) and decreases along the parts' thickness direction. The concentration profile is obtained by combining the evaporation and mass flow exiting the domain through the bottom surface (right edge in the figure).

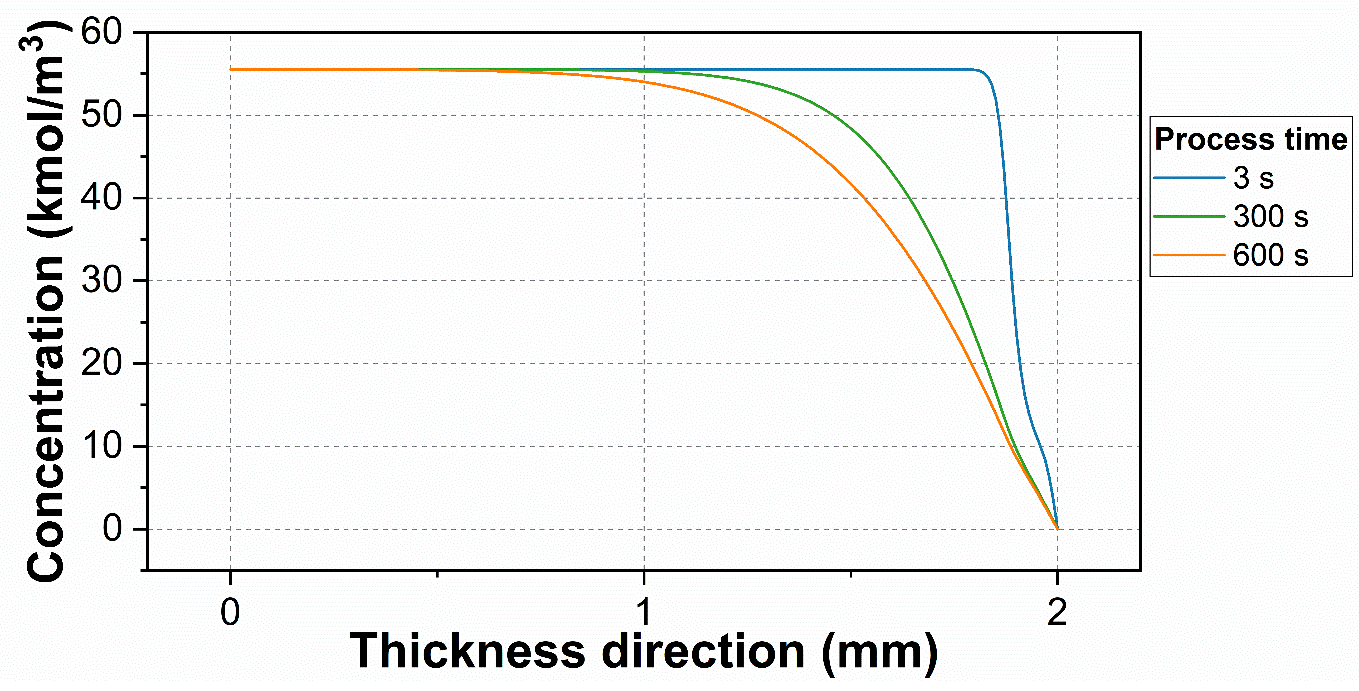

Figure 17: Simulated water concentration profile at times $3 \mathrm{~s}, 300 \mathrm{~s}$ and $600 \mathrm{~s}$ along the parts' thickness direction.

Figure 20 shows the simulated and experimentally calculated cold pressing dryness efficiency for the different processing conditions investigated. The percentage difference in prediction varies from a minimum of $0.3 \%$ to a maximum of $25.8 \%$. A particular point 
to note while assessing the accuracy of the simulations is that the measured thicknesses before $\&$ after the cold pressing of moulded pulp were inputs to the numerical model. For one of the three cases for the $0.6 \mathrm{MPa} 3 \mathrm{~s}$ cold pressing, the starting thickness was significantly different than the other two cases ( $>10 \%$ thickness difference) indicating a different initial condition with respect to both water content and material properties, that resulted in an outlier prediction with $\sim 14 \%$ deviation from experimental measurement. For the other two cases, the difference of prediction in dryness efficiency was $0.4 \%$ and $1.3 \%$, indicating a very good model prediction capability. Excluding the abovementioned outlier, the maximum difference between experiments and simulation was $<5 \%$ for the $3 \mathrm{~s}$ and $300 \mathrm{~s}$ time durations. Similarly, differences between the initial conditions for the $600 \mathrm{~s}$ experiments with respect to the $3 \mathrm{~s}$ and $300 \mathrm{~s}$ experiments, had an influence on the accuracy of the simulation results for the $600 \mathrm{~s}$ cases. 


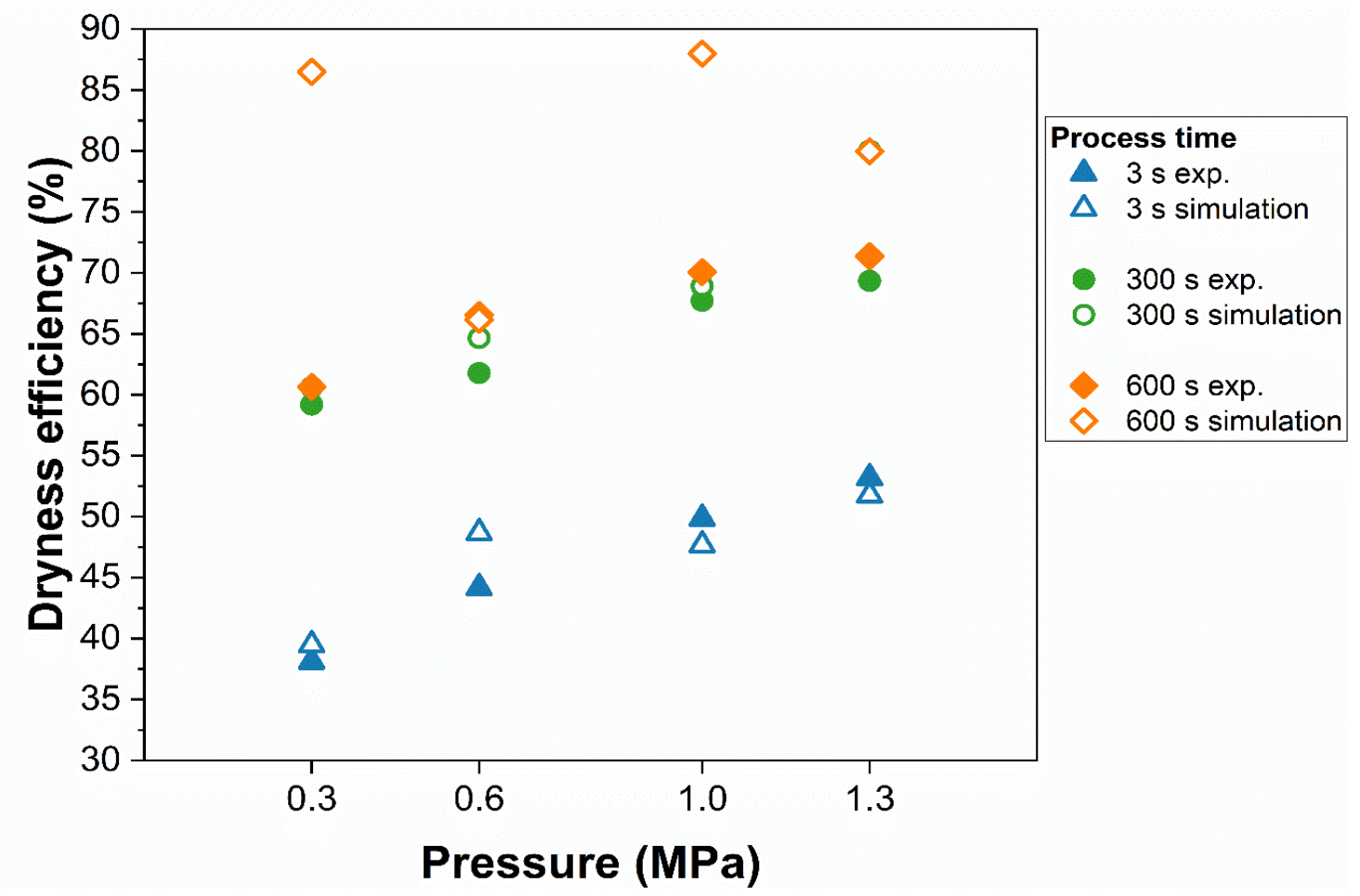

Figure 18: Experimental and simulated results for the dryness efficiency as a function of increasing pressure and process time for the cold pressing experiments.

In Figure 21, the simulations of the dewatering during thermoforming are shown and compared with the experimental results. In this case, the percentage difference in prediction varies from a minimum of $0.3 \%$ to a maximum of $6.4 \%$. It was not possible to predict the dewatering for the case at $160{ }^{\circ} \mathrm{C}$ because vaporization of the water content occurred within the sample domain, thus violating one of the model assumptions. For the just mentioned case, the simulation returned a null water concentration, which indicates the creation of a vapour front or bubble. 


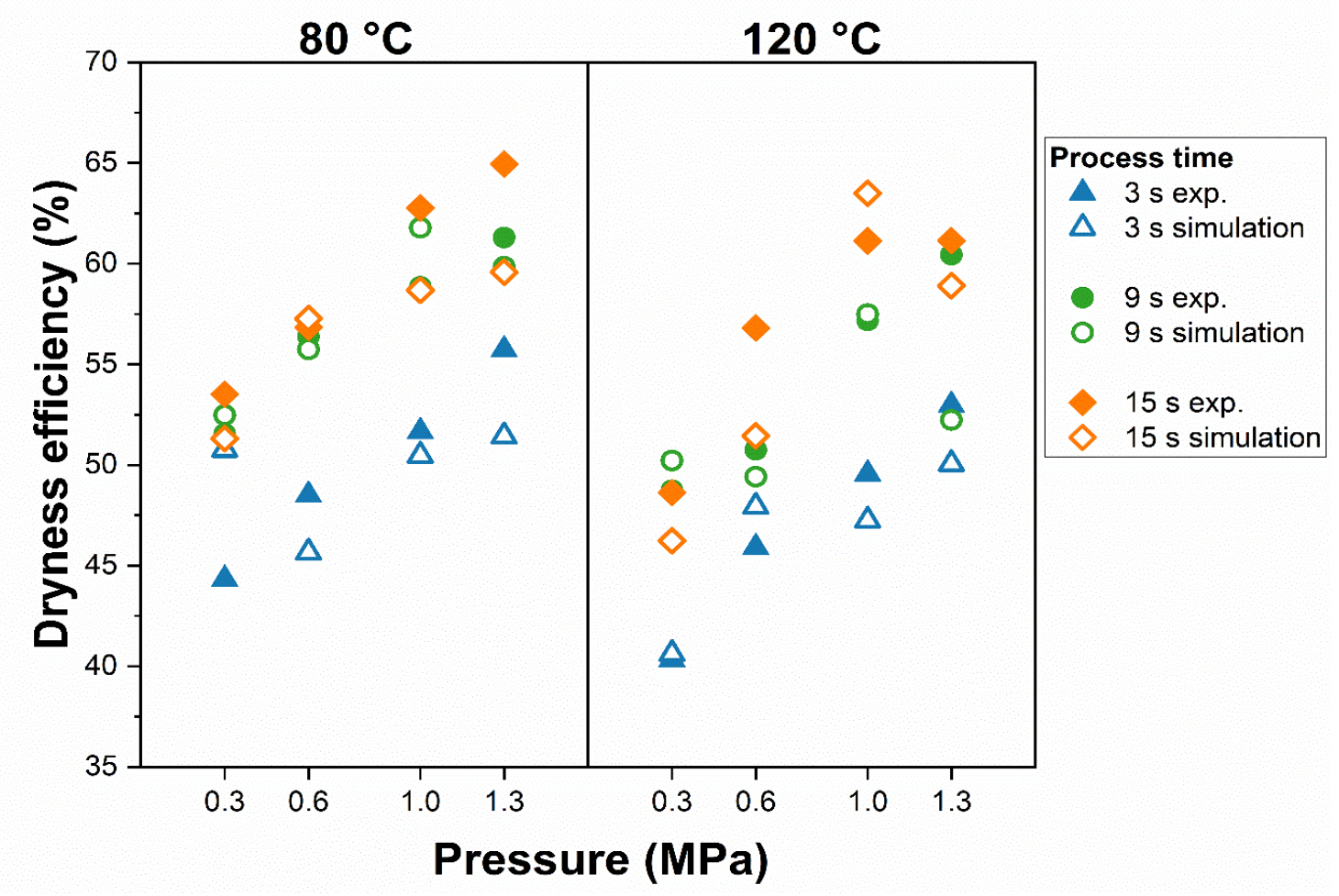

Figure 19: Experimental and simulated results for the dryness efficiency as a function of increasing pressure, temperature and process time for the thermoforming experiments.

\section{Discussion}

As mentioned before the first source of variance and error in the simulations is due to the values used as initial conditions: initial and bone-dry sample weight and initial sample's thickness. These measured values present large variations, which are then reflected on the calculated value for porosity (and correspondingly water removal) over time.

The thermo-forming simulations give accurate results, with a difference in dryness efficiency prediction down to $0.3 \%$, when the process time is relatively short, up to $15 \mathrm{~s}$, and the thickness variation is small, up to $60 \%$. On the other hand, the results get less coherent with experimental measurements when the time and the thickness variation increases, with a difference in prediction up to $25.8 \%$. The limitations of the current model can be conceived to arise out of the following: 
i) The model assumes homogeneous material properties in all the considered cases while only changing the geometry based on experimental measurements. In reality, the measured values for thickness, initial and bonedry weight widely differ across the experiments (variation as large as $10 \%$ ) and correspond to different effective material properties of the pulp.

ii) The simulations did not consider vaporization (by cavitation/boiling) within the domain, as well as the changing of pressure conditions due to the continuous crushing of the pulp fibres. Thus, cases corresponding to large changes in dimensions within the simulated period can result in greater simulation mis-prediction.

iii) Only a two phase system (of wood \& water) was considered instead of a three phase system including the gas-filled pores. The latter would have required implementation of Richards' equation (that adds a pore volume-fill fraction to the Darcy flow equation, and which has known instability and convergence issues) instead of the current simpler Darcy-Forchheimer model.

iv) The specific-surface-area term of the Kozeny-Carman equation (equation (7) and (8)) is based on the assumption of an impermeable solid. However, wood fibres are highly permeable.

Concluding, the partially decoupled one-dimensional model presented here offers good fast predictions for the dryness efficiency in the case that the part deformation is not relatively large and within shorter process times. The presented model is the first model in literature for the thermo-forming process and can be naturally extended to a more complete two or three-dimensional model, also taking into account the effect of poroelasticity, the Richards' equation [37] (i.e. the movement of water in unsaturated porous medium) and bound-moisture-dependent material properties. The creation of a 
vapour front by cavitation within the part domain could also be considered. Delamination could then be indirectly modelled by setting a vapour concentration threshold, above which delamination occurs.

\section{Conclusions}

The present paper documents the thermo-forming process for manufacturing moulded paper products. Experimental investigations show the dependence of the process output (i.e. dryness efficiency) on the three chosen process parameters of pressure, temperature and holding time. The experiments also form the basis for setting up a multi-physics model of the process that can be used to predict and control process parameters for achieving optimum dryness in the moulded products.

The current implementation of the model describes the dewatering phenomena during a thermoforming process in the production of moulded pulp products, by considering a combination of direct dewatering due to volume reduction by compression, the flow of water through the porous medium (i.e. Darcy flow) and evaporation due to heat transfer from the pressing piston.

The simulation gives accurate estimates, with a difference in prediction down to $0.3 \%$, when the considered process time is short (up to $15 \mathrm{~s}$ ) and the relative thickness reduction small (up to $60 \%$ ). The applicability of the simulation towards widely varying initial conditions \& long modelled duration has also been discussed in more detail.

Because of the non-linear nature of the equations, it is not possible to generalize the model for any type of pulp grades and processing conditions without a method to estimate the right material properties. However, the general approach and experimental methodologies presented should be applicable to any specific production of moulded pulp product. 
The extension to a more complete two or three-dimensional model, which takes into account poroelasticity effects, the creation of a vapour front and moisture-dependent material properties, should be a topic deserving further study.

In summary, the current paper presents the first modelling study on thermoforming of moulded pulp products, provides validation against experimental observations, discusses in succinct the strength and applicability of the simple model as well as the natural extensions of the model to obtain greater accuracy.

\section{Acknowledgement}

This work was supported by the Innovation Fund Denmark under Grant number 510600006B.

\section{Nomenclature}

$\begin{array}{lll}\dot{m}_{\text {evap }} & \text { Specific moisture evaporation rate } & \mathrm{kg} /\left(\mathrm{m}^{3} \mathrm{~s}\right) \\ A_{p} & \text { Specific permeability } & \mathrm{g} / \mathrm{m} \\ C_{p} & \text { Specific heat capacity } & \mathrm{J} /(\mathrm{kg} \mathrm{K}) \\ D_{e} & \text { Dryness efficiency } & \\ L_{\text {evap }} & \text { Latent heat of vaporization } & \mathrm{J} / \mathrm{kg} \\ T H K_{\text {in }} & \text { Initial thickness } & \mathrm{m} \\ T H K_{\text {out }} & \text { Final thickness } & \mathrm{m} \\ T H K_{\text {red }} & \text { Thickness reduction } & \% \\ k_{f} & \text { Fibre thermal conductivity } & \mathrm{W} /(\mathrm{m} \mathrm{K}) \\ k_{i} & \text { Inertial permeability } & \\ k_{\text {par }}(T, p) & \text { Parallel thermal conductivity } & \mathrm{W} /(\mathrm{m} \mathrm{K}) \\ k_{\text {ser }}(T, p) & \text { Series thermal conductivity } & \mathrm{W} /(\mathrm{m} \mathrm{K}) \\ k_{w}(T, p) & \text { Water thermal conductivity } & \mathrm{W} /(\mathrm{m} \mathrm{K}) \\ m_{0} & \text { Initial moisture content } & \\ m_{e} & \text { Limiting (or equilibrium) moisture content } & \\ m_{f} & \text { Sample bone dried weight } & \mathrm{kg} \\ m_{\text {in }} & \text { Initial sample weight } & \mathrm{g} \\ m_{\text {out }} & \text { Final sample weight } & \mathrm{g} \\ \varepsilon_{f} & \text { Fibre volume fraction } & \\ \varepsilon_{w} & \text { Water volume fraction } & \mathrm{m}{ }^{2} \\ \kappa_{i} & \text { Flow inertial permeability } & \mathrm{m}{ }^{2} \\ \kappa_{p} & \text { Permeability of the porous medium } & \mathrm{kg} / \mathrm{m}^{3} \\ \rho_{\text {water }} & \text { Water density } & \\ \sigma_{c} & \text { Combined standard deviation } & \\ \epsilon_{p} & \text { Porosity of the moulded pulp } & \end{array}$




$\begin{array}{lll}\nabla p & \text { Pressure gradient } & \mathrm{Pa} \\ C 1 & \text { Estimated proportionality constant } & \\ A & \text { Area of the surface } & \mathrm{m}^{2} \\ \text { Are } a_{\text {disk }} & \text { Sample area } & \mathrm{m}^{2} \\ D P & \text { Decreasing Permeability } & \\ I & \text { Press impulse } & \mathrm{kPa} \mathrm{s} \\ N B S K & \text { Nordic Bleached Softwood Kraft } & \\ S & \text { Consistency } & \% \\ S E M & \text { Scanning Electron Microscope } & \\ T & \text { Temperature } & { }^{\circ} \mathrm{C} \\ W & \text { Basis weight } & \mathrm{kg} / \mathrm{m}^{2} \\ a & \text { Empirical parameter } & \\ k & \text { Thermal conductivity } & \mathrm{W} /(\mathrm{m} \mathrm{K}) \\ k & \text { Thermal conductivity } & \mathrm{W} /(\mathrm{m} \mathrm{K}) \\ m & \text { Final moisture content } & \\ n & \text { Compressibility factor } & \\ q & \text { Diffusive heat flow perpendicular to the surface of area } & \mathrm{W} \\ u & \text { Flow velocity through the porous medium } & \mathrm{m} / \mathrm{s} \\ x & \text { Space parameter } & \mathrm{m} \\ \mu & \text { Water viscosity } & \mathrm{kg} /(\mathrm{m} \mathrm{s}) \\ v & \text { Kinematic viscosity } & \mathrm{m} 2 / \mathrm{s} \\ \rho & \text { Density } & \mathrm{kg} / \mathrm{m}^{3}\end{array}$

\section{References}

(1) Didone, M.; Saxena, P.; Brilhuis-Meijer, E.; Tosello, G.; Bissacco, G.; McAloone, T. C.; Pigosso, D. C. A.; Howard, T. J. Moulded Pulp Manufacturing: Overview and Prospects for the Process Technology. Packag. Technol. Sci. 2017, 30 (6), 231-249. https://doi.org/10.1002/pts.2289.

(2) Saxena, P.; Bissacco, G.; Bedka, F. J.; Stolfi, A. Tooling for Production of the Green Fiber Bottle. Procedia CIRP 2018, 69, 348-353. https://doi.org/10.1016/j.procir.2017.12.001.

(3) Roux, J. C.; Vincent, P. A Proposed Model in the Analysis of Wet Pressing. Tappi J. 1991, 74 (2), 189-195.

(4) Lee, C. A.; Furbeck, W. R.; Granberg, F. M. Method and Apparatus for Making a Molded Pulp Product with a Liquid Impervious Layer Integrally Secured Thereon. US3957558A, April 19, 1974. 
(5) Skjelmose, F. I. Method for the Manufacturing of a Moulded Body. US005997785A, 1999.

(6) Juul, N. Method of Producing Moulded Pulp Articles with a High Content of Dry Matter. US6517684B1, 2003.

(7) Björn, N.; Graffton, L.; Baskman, L. Pulp Mould and Use of Pulp Mould. US7909964B2, 2005.

(8) Michell, A. J. Press Drying of Paper - an Overview. Appita 1984, 37 (4).

(9) Stenström, S. Drying of Paper: A Review 2000-2018. Dry. Technol. 2019, 0 (0), 1-21. https://doi.org/10.1080/07373937.2019.1596949.

(10) Tappi. Forming Handsheets for Physical Tests of Pulp - T205. 1995, pp 1-9.

(11) Kerekes, R. J.; McDonald J. D. A Decreasing Permeability Model of Wet Pressing - Theory. Tappi J. 1991, 74 (12), 150-156.

(12) Khiabani, R. H.; Joshi, Y.; Aidun, C. K. Heat Transfer in Hot Pressing of Saturated Fiber Suspensions. J. Therm. Sci. Eng. Appl. 2010, 2 (4), 041009. https://doi.org/10.1115/1.4003509.

(13) McDonald, D. J.; Kerekes, R. J. Estimating Limits of Wet Pressing on Paper Machines. Tappi J. 2017, 16 (2), 81-87.

(14) Walker, J. Boiling and the Leidenfrost effect https://www.wiley.com/college/phy/halliday320005/pdf/leidenfrost_essay.pdf (accessed Sep 20, 2018).

(15) Zavaglia, J. C.; Lindsay, J. D. Flash X-Ray Visualization of Multiphase Flow during Impulse Drying. Tappi J. 1989, 79-85.

(16) Macklem, E. A. Impulse Drying - a Pressing/Flashing Drying Phenomena. In Engineering Conference, Proceedings of the Technical Association of the Pulp and Paper Industry; 1988. 
(17) JCGM. Evaluation of Measurement Data — Guide to the Expression of Uncertainty in Measurement. International Organization for Standardization Geneva. 2008, p 134. https://doi.org/10.1373/clinchem.2003.030528.

(18) Ramaswamy, S. Vacuum Dewatering During Paper Manufacturing. Dry. Technol. 2003, 21 (4), 685-717. https://doi.org/10.1081/DRT-120019058.

(19) Lobosco, V.; Kaul, V. The Stress-Strain Relationship of the Fibre Network in Wet Pressing. Nord. Pulp Pap. Res. J. 2005, 20 (01), 024-029. https://doi.org/10.3183/NPPRJ-2005-20-01-p024-029.

(20) El-Hosseiny, F. Mathematical Modeling of Wet Pressing of Paper - A Review of the Literature. Nord. Pulp Pap. Res. J. 1991, 06 (01), 030-034. https://doi.org/10.3183/NPPRJ-1991-06-01-p030-034.

(21) Hewitt, D. R.; Paterson, D. T.; Balmforth, N. J.; Martinez, D. M. Dewatering of Fibre Suspensions by Pressure Filtration. Phys. Fluids 2016, 28 (6), 063304. https://doi.org/10.1063/1.4952582.

(22) Ratto, P.; Rigdahl, M. The Deformation Behaviour in the Thickness Direction of Paper Subjected to a Short Pressure Pulse. Nord. Pulp Pap. Res. J. 1998, 13 (03), 180-185. https://doi.org/10.3183/NPPRJ-1998-13-03-p180-185.

(23) Szokla, Z.; Paulapuro, H. Changes in Z-Direction Density Distribution of Paper in Wet Pressing. In 1987 International Paper Physics Conference; TAPPI, 1987; pp $113-119$.

(24) He, J.; Batchelor, W. J.; Johnston, R. E. The Behavior of Fibers in Wet Pressing. Tappi J. 2003, 2 (12), 27-31.

(25) Back, E. L. Steam Boxes in Press Sections - Possibilities and Limitations. Appita $J .1988,41(3)$.

(26) Bloch, J. F.; Auriault, J. L. Physics Modelling from Microscale to Macroscale: 
Homogenisation Technique. Application to Hot Pressing of Paper. In Polymers, Laminations, and Coatings Conference; Tappi Press, 1999; pp 381-390.

(27) Nilsson, L. S.; Stenstrom, S. G. Modelling the Effective Mass and Heat Diffusivities of Pulp and Uncoated Paper. Am. Soc. Mech. Eng. Heat Transf. Div. Htd 1995, 317 (2), 421-426.

(28) Rudman, I.; Orloff, D. I. Dynamic Thickness and Temperature Measurements during Wet Pressing and Impulse Drying. Tappi J. 2002, 1 (5), 8-12.

(29) Wyllie, M. R. J.; Gardner, G. H. F. Generalized Kozeny-Carman Equation. World Oil 1958, $146(5)$.

(30) McDonald, J. D.; Kerekes, R. J. Pragmatic Mathematical Models of Wet Pressing in Papermaking. Bioresources 2017, $12 \quad$ (4), 9520-9537. https://doi.org/10.15376/biores.12.4.McDonald.

(31) Hattel, J. Fundamentals of Numerical Modelling of Casting Processes; Polyteknisk Forlag, 2005.

(32) Nederveen, C. J.; Finken, J. G. M. Thermal Conductivity Measurements on Wet Paper Samples at High Temperatures. Dry. Technol. 1992, 10 (1), 189-198.

(33) Palosaari, S. M.; Cornish, A. R. H. Approximate Method for the Prediction of the Effective Thermal Conductivity of Wetter Porous Materials. Acta Polytech Scand Chem Incl Met. Ser Ch 1976.

(34) Gray, W. G.; Miller, C. T. Examination of Darcy's Law for Flow in Porous Media with Variable Porosity †. Environ. Sci. Technol. 2004, 38 (22), 5895-5901. https://doi.org/10.1021/es049728w.

(35) De Schampheleire, S.; De Kerpel, K.; Ameel, B.; De Jaeger, P.; Bagci, O.; De Paepe, M. A Discussion on the Interpretation of the Darcy Equation in Case of Open-Cell Metal Foam Based on Numerical Simulations. Materials (Basel). 2016, 
9 (6), 409. https://doi.org/10.3390/ma9060409.

(36) Nilsson, J.; Stenström, S. Modelling of Heat Transfer in Hot Pressing and Impulse Drying of Paper. Dry. Technol. 2001, 19 (10), 2469-2485. https://doi.org/10.1081/DRT-100108249.

(37) Richards, L. A. Capillary Conduction of Liquids through Porous Mediums. Physics (College. Park. Md). 1931, $1 \quad$ (5), 318-333. https://doi.org/10.1063/1.1745010. 